\title{
Dual Mode Control Strategy for the Energy Efficiency of Complex and Flexible Manufacturing Systems
}

\author{
Jenny L. Diaz C. ${ }^{a}$, Carlos Ocampo-Martinez ${ }^{\mathrm{a}}$, Sorin Olaru ${ }^{\mathrm{b}}$ \\ ${ }^{a}$ Automatic Control Department, Universitat Politècnica de Catalunya, Institut de Robòtica i Informàtica Industrial (CSIC-UPC), Llorens i Artigas 4-6, Planta 2, \\ 08028 Barcelona, Spain (e-mail: \{jenny.lorena.diaz, carlos.ocampo\}@upc.edu. \\ ${ }^{b}$ Laboratory of Signals and Systems (L2S, UMR CNRS 8506), Centrale Supélec-CNRS Université Paris Sud, Université Paris-Saclay, 91190 Gif-sur-Yvette, France \\ (e-mail: sorin.olaru@centralesupelec.fr).
}

\begin{abstract}
The manufacturing industry is shifting towards smart manufacturing, in which both energy efficiency and flexibility are some of the main objectives of this digital transformation. In this regard, the control strategies for manufacturing systems should be able to support the requirements of this transformation with a low computational burden towards their implementation in real time. To this end, in this paper, a dual mode control strategy based on two control approaches is proposed to minimise the energy consumption of manufacturing systems without affecting their productivity, even when scenarios of flexible manufacturing are considered. The first control mode is based on model predictive control to determine an optimisation-based strategy for the constrained behaviour of the system. Then, the second mode builds on the assumption that the system exhibits a periodic behaviour and, thus, it will be able to switch to an autonomous control mode that avoids the resolution of an optimisation problem online. The proposed control strategy is tested in a manufacturing process line in which changes in the production programs are considered with the aim to test the performance in flexible manufacturing scenarios. The obtained results show that the computational burden could be significantly reduced while reducing global energy consumption without affecting the system productivity.
\end{abstract}

Keywords: Dual mode control strategy, Model predictive control, Mixed-integer linear programming, Smart manufacturing systems, Flexible manufacturing

\section{Introduction}

Manufacturing refers to the industrial production processes through which the raw materials are transformed into finished products to be sold in the market. Thus, manufacturing systems can be considered as a set of processes for transforming resources and energy into industrial products and goods for consumers [5, 18]. Since manufacturing systems consume most of the electricity produced in the world, the minimisation of the energy consumption of such systems does not only allow reducing the energy costs of a manufacturing plant but also contributes to minimising the global electricity demand. Thus, due to the depletion of fossil energy sources, rising energy prices, and governmental regulation restrictions, the current manufacturing industry is shifting towards more efficient and sustainable systems. This fact has promoted the identification of energysaving opportunities and the design of strategies oriented to improve the energy efficiency of such systems [19]. Besides, into the new era of manufacturing systems and their transformation towards smart manufacturing, these systems should be able to respond to changes in the production programs to processing pieces with different design specifications, allowing a higher level of customizability for the final users [3].

In this regard, manufacturing systems in common acceptance is referred to as a collection of machines and devices organised in a proper configuration for producing a finished piece, com- monly named as process line. Complex structures are found in a process line, which corresponds to the aggregation of machines, peripheral devices, and buffer devices for producing a piece. At this aggregation level, machines are organised logically according to the required operations to process a piece entirely. Besides, since the machines in a process line could have different operating cycles, buffers might be required in order to maximise the production and avoid the simultaneous input of two or more pieces to one machine. In these systems, peripheral devices refer to those devices that are not directly related to machining operations but are necessary to guarantee the correct operation of machines and the suitable supply of resources to machines in the process line. Some resources required by machines are, for instance, water to clean pieces, the coolant to be used during machining operations, air or hydraulic fluids to clamp/unclamp of pieces according to the design of the machine, among others.

According to the processes performed on each machine and its connections, process lines present different configurations, such as serial and parallel structures. Based on the configurations in a process line, different energy and material flows, which represent either the interactions or relations between the machines and peripheral devices in the process line, could exist. These relationships add complexity and represent a barrier for the understanding and modelling of the energy consumption as well as for designing control/management strategies at this level. Therefore, factors as the diversity of components in 


\section{Nomenclature}

\section{Physics Constants}

$\rho_{c} \quad$ Density of coolant

$R \quad$ The gas constant

$T \quad$ Temperature of air in $T_{1}$

$W_{\text {air }} \quad$ The molecular weight of air

Number Sets

$\mathbb{R} \quad$ Real number set

$\mathbb{R}_{>0} \quad$ Set of positive real number

$\mathbb{V}_{2} \quad$ Discrete domain of $P_{G, 2}$

$\mathbb{Z} \quad$ Integer number set

$\mathbb{Z}_{\geq 0} \quad$ Set of positive integer number

Acronyms

LTI Linear time-invariant

MC Markov chains

MPC Model predictive control

PN Petri nets

SI Subspace identification

\section{Symbols}

$\alpha_{v_{1}} \quad$ Constant energy consumption of valve $v_{1} 100 \%$ open

$\alpha_{v_{3}} \quad$ Constant energy consumption of valve $v_{3} 100 \%$ open

$\beta_{i} \quad$ Integral of the Energy consumption of $M_{i}$ along $T_{M_{i}}$

$\eta \quad$ Pump efficiency

$\gamma_{P_{j}} \quad$ State vector of the energy consumption model of $P_{j}$

$\Gamma^{\Gamma} \quad$ Sequence of $\boldsymbol{\Lambda}_{\mathbf{P}}$ along $H_{p}$

$\Lambda_{\mathbf{P}} \quad$ The activation sequence of peripheral devices in the process line

$\boldsymbol{\Lambda}_{\mathbf{M}_{\mathbf{i}}}$ The activation sequence of devices of the $i$-th machine

$\Lambda_{\mathrm{P}}^{*} \quad$ Optimal activation sequence of peripheral devices

$\Lambda_{\mathbf{v}}^{*} \quad$ Optimal activation sequence of valve aperture

$\mathbf{D}_{\mathbf{p k}}$ Distance vector among the first lag and the rest of the lags in lc

Ic Vector of lags among the peaks of $\mathbf{u x}_{\mathbf{j}}$

pk Peaks of in vector $\mathbf{u x}_{\mathbf{j}}$

$\mathbf{u x}_{\mathbf{j}} \quad$ Vector of autocorrelation coefficients of signal $u_{j}$

$\bar{q}_{G_{1}} \quad$ Upper bound for process dynamics related to $P_{G_{1}}$

$\bar{q}_{G_{2}} \quad$ Upper bound for process dynamics related to $P_{G_{2}}$

$\bar{q}_{L_{2}} \quad$ Upper bound for process dynamics related to $P_{L_{1}}$

$\frac{q_{L_{1}}}{u x_{j}} \quad$ Maximum autocorrelation coefficient of signal $u_{j}$

$\tau_{s} \quad$ Sampling time

$\underline{q}_{G_{1}} \quad$ Lower bound for process dynamics related to $P_{G_{1}}$

$\underline{q}_{G_{2}} \quad$ Lower bound for process dynamics related to $P_{G_{2}}$

$\underline{q}_{L_{1}} \quad$ Lower bound for process dynamics related to $P_{L_{1}}$

$\frac{q_{L}}{\varepsilon_{M_{1}}} \quad$ Maximum flow required by $M_{1}$

$A_{T_{2}} \quad$ Transverse area of $T_{2}$

$A_{T_{3}} \quad$ Transverse area of $T_{3}$

$A p_{v_{1}} \quad$ Aperture of valve $v_{1}$

$A p_{v_{3}} \quad$ Aperture of valve $v_{3}$ $b \quad$ Number of machines in the process line

$H_{p} \quad$ Prediction horizon

$h_{f_{1 \rightarrow 2}} \quad$ Energy losses by friction

$H_{p, c} \quad$ Prediction horizon in the commutation protocol

$J \quad$ Integral of the energy consumption signal

$k \quad$ Discrete-time index

$L_{2} \quad$ Level of clean coolant in $T_{2}$

$L_{3} \quad$ Level of dirty coolant in $T_{3}$

$m \quad$ Number of manufacturing devices in each machine

$m_{c} \quad$ Flow of dirty coolant pumped by $P_{G, 2}$

$M_{T_{1}} \quad$ Mass of air in the tank 1

$M_{T_{4}} \quad$ Mass of air in the tank 4

$m_{c c l, M_{i}} \quad$ Flow of clean coolant required by $M_{i}$

$m_{d c l, M_{i}} \quad$ Flow of dirty coolant recovered from $M_{i}$

$N \quad$ Model order

$n \quad$ Number of peripheral devices in the process line

$N_{u} \quad$ Time to start running periodicity detection

$p \quad$ Dimension of the state vector $\gamma_{P_{j}}$

$P_{G_{1}} \quad$ Global peripheral device 1

$P_{G_{2}} \quad$ Global peripheral device 2

$P_{i n} \quad$ Input pressure in the pipe system

$P_{L_{1}} \quad$ Local peripheral device 1

$P_{\text {out }} \quad$ Output pressure in the pipe system

$P_{T_{1}} \quad$ Pressure of air inside the tank 1

$P_{T_{4}} \quad$ Pressure of air inside the tank 4

$q_{P_{j}} \quad$ States for the dynamics of $P_{j}$

$r \quad$ Dimension of the state vector $\xi_{M_{i, 1}}$

$S \quad$ Total energy consumption of the process line

$S_{M_{i}} \quad$ Power consumption of the $i$-th machine

$S_{P_{j}} \quad$ Power consumption of the $j$-th peripheral device

$S_{v_{1}} \quad$ Energy consumption of valve $v_{1}$

$S_{v_{3}} \quad$ Energy consumption of valve $v_{3}$

$T_{2} \quad$ Tank with clean coolant

$T_{3} \quad$ Tank with dirty coolant

$T_{M_{i}} \quad$ Cycle time of the $i$-th machine

$T_{u_{j}} \quad$ Period of the peripheral device $j$

$u_{M_{i, l}} \quad$ Activation/deactivation signal of the $l$-th device of the $i$-th machine

$u_{P_{j}} \quad$ Activation/deactivation signal of the $j$-th peripheral device

$V_{T_{1}} \quad$ Volume of $T_{1}$

$V_{T_{4}} \quad$ Volume of $T_{4}$

J $\quad$ Sequence for control objective $J$ along $H_{p}$

$\omega \quad$ Specific work per time unit

$M_{i} \quad$ The $i$-th machine

$m_{\text {air }, M_{i}} \quad$ Consumption of air from machine $M_{i}$

$m_{\text {in }} \quad$ Air flow pumped by $P_{G}$

$m_{G, 1_{M_{1}}} \quad$ Flow of air provide by $P_{G_{1}}$ to machine 1

$m_{L, 1_{1}} \quad$ Flow of air provide by $P_{L_{1}}$ to machine 1 a process line, the pattern of their energy consumption, their interactions, and the intrinsic characteristics of each component should be considered to analyse and propose energy efficiency improvements at the process-line level [4]. However, to reduce the energy consumption of manufacturing systems, the improvements for each and single machine or device in the process line are not enough. Instead, the whole process line should be optimised to reduce its total energy consumption [15]. According to this fact, most of the strategies and technologies identified from literature are focused on capturing and tracking energy and material flows in a multi-machine ecosystem, and the offline processes planning and scheduling of machines.

Process planning and scheduling are essential (and complementary) factors regarding energy consumption and flexibility of manufacturing systems. The currently implemented strategies in manufacturing systems have only focused on the development of adaptable and sustainable processes plans, which can be flexible regarding the market requirements. In [2], an approach of integration between the Energy-Aware Scheduling (EAS) methodology and a reference schedule generated by an Advanced Planning and Scheduling (APS) system that does not consider energy saving is proposed. This approach is employed together with a process model to control the power peaks in the shop floor for a given detailed schedule. Similarly, in [9], the energy-efficient Permutation Flow-shop Scheduling Problem with Controllable Time (PFSPCT) is studied to analyse the scheduling problem but including both transportation and processing times. A different approach that incorporates power 
models for a single machine and cutting-parameters optimisation into the scheduling problems is developed in [17]. In this last reference, the scheduling problem is solved by using a multi-level optimisation approach considering both machine and line levels.

In the same context, the control of peak power demand has been another research focus towards the improvement of the energy efficiency for manufacturing systems at the process line level. In this sense, most of the strategies currently implemented regarding the peak power control consist of delaying the switching-on of some machines taking into account a given threshold value. Usually, this latter value corresponds to the contracted nominal power, which produces economic penalties if exceeded. Although many works consider strategies for flexible manufacturing at the plant level, energy consumption is usually included as an off-line optimisation regarding the production planning of the existing devices in the plant. That is, these strategies determine an optimal sequence from the beginning, and therefore they cannot respond to the real-time variation of processes and working environment factors during the operation of the plant [3].

Given the complexity of manufacturing systems as a function of the processes performed, the strong relationships between the peripheral and machining devices, the time-varying constraints such as tool wear, the efficiency of each device and the changing-working environment, the most used control techniques in manufacturing systems are those based on optimisation. This conclusion comes from the fact that the control objectives, operating constraints of either devices or machines involved, their operating ranges, dynamic expressions for the relationships between machines and their environment, and any additional constraints that condition the performance of the system to be controlled can be included into an optimisation problem. However, although few control applications in manufacturing systems consider energy objectives, most of them have been limited to analyse the individual system and not consider interactions with both other devices and machines. Besides, most of these applications consist of designing closed-loop control schemes that minimise the difference between the real energy consumption and a reference behaviour, which is usually determined offline and without considering the temporal variations of its surroundings. Thus, in most of the case studies, disturbances or changes in the working environment are not considered. Therefore, and taking into account the current context of manufacturing industry, strategies able to respond in real time to any changes in the system or its environment, besides to consider flexibility in the processes plan and schedule should be developed [1].

In this paper, a dual-mode control strategy based on two control approaches is proposed to minimise the energy consumption of manufacturing systems without affecting their productivity. The main idea behind the design of the dual control strategy is to reduce the computational burden by switching from a control mode based on on-line optimisation to an autonomous mode without real-time optimisation. The latter is a built-in control strategy that can be implemented in real time, considering both flexible manufacturing scenarios and disturbances management, but without sacrificing the performance of the controller in closed loop. To this end, the first control mode or the predictive one is designed based on Model Predictive Control (MPC) to determine the optimal system behaviour taking into account the current state of the process line (machines and peripheral devices), the production program currently executed, and the operational and physical constrains of both machines and peripheral devices into the process line. Then, the second mode starts from the assumption that the system could exhibit periodic behaviour, inherited from the periodic behaviour of the machines. Therefore, when the system periodicity is detected the control system switches to an autonomous control mode. In this latter mode, the optimal and periodic behaviour achieved in the first control mode as result of the optimisation is repeated over time. However, to avoid undesirable behaviour of the process line, a prediction and a commutation protocol in which it is verified that the system remains into its feasible domain are performed while the autonomous mode is running. The proposed approach is tested on a benchmark problem to minimise the global energy consumption of a serial process line with three machines and three peripheral devices, taking into account disturbances in the operation of peripheral devices and flexibility scenarios such as changes in the production programs.

The remainder of the paper is organised as follows. In Section 2, the problem statement considered in this paper is presented. Next, Section 3 introduces the case study and present the way to model both energy consumption and process dynamics of peripheral devices and machines in a process line. In Section 4, the proposed control strategy and the two control modes are presented and described. Besides, in Sections 4.1 and 4.2 , the algorithm for detecting periodicity and the commutation protocol are explained, respectively. Afterwards, obtained results from the implementation of the proposed control strategy and an analysis of its effectiveness in disturbance scenarios are reported and discussed in Section 5. Finally, in Section 6 , conclusions and future works based on the obtained results are drawn.

\section{Problem Statement}

The main units in a manufacturing systems are the machine tools, which can be understood as a set of different devices that work in a sequential way to process a piece during a fixed period of time. Most of these machines are organised in a logical and sequential way to process a piece completely, which shapes a process line. In the current context of flexible manufacturing, process lines should be able to produce different finished parts, in which the line has the flexibility to react to changes in the product being manufactured, both in type and quantity. In this regard, a process line is a complex (and large-scale) system including several machines and peripheral devices that work synchronously and logically up to getting a finished part. Machines in a process line $\left(M_{i}\right)$ correspond to a set of devices that are directly related to machining processes (e.g., milling, cutting, turning, grinding, drilling), while the peripheral devices are those devices that provide the resources required to machines for their proper operation. 
The machines in a process line are characterised by a periodic behaviour according to the total time required for manufacturing a piece, which corresponds to a operation cycle denoted here by $T_{M_{i}}$. Therefore, the energy consumption of devices directly related to manufacturing operations in each machine, such as manufacturing processes, transport, and handle of pieces, shows also a periodic behaviour. However, due to the nature of the operations performed by machine devices (e.g., rotational motions, axial motions, cutting, milling), there exist stages of both high and low energy consumption along $T_{M_{i}}$.

On the other hand, since peripheral devices supply resources to machines in the process line, there exist several functional relationships between machines and peripheral devices that determine the productivity of the process line. When the machines in a process line have different values of $T_{M_{i}}$, the throughput of the process line level is determined based on the machine with the longest cycle time $\max \left(T_{M_{i}}\right)$. Thereby, process lines are designed with the aim of processing a complete piece up to achieve the desired physical properties (e.g., shape, weight, volume, surface, among other) in the shortest possible time. However, peripheral devices might or might not show a periodic behaviour, which may match with $T_{M_{i}}$, as a consequence of the design of peripheral devices in the process line as well as the way they are managed. Therefore, to improve the energy efficiency of a process line, peripheral devices must be correctly managed such that their activation time does not match with the time instants/slots of higher consumption while satisfying the operating constraints of machines.

At the process line level, the functional relationships between machines and peripheral devices could be quite complex since some peripheral devices can be shared between two or more machines, provide the same resource to all the machines in the line, or even supply the same resource provided by another global peripheral device to a particular machine. Besides, peripheral devices in the process line should be able to adapt their operation to the changes in the production program and the new functional relationships imposed by these programs. In these cases, a consensus based on the management objectives (e.g., energy consumption, energy costs, productivity) should be established to guarantee the satisfaction of the operating constraints among machines and peripheral devices as well as the proposed control objectives. In Figure 1, a serial process line is shown, which consists of three machines and three peripheral devices. Based on the configuration of peripheral devices in the process line, they can be classified as:

- Global peripheral device if it is shared among two or more machines of the process line, and

- Local peripheral device of the machine $i$ when the device works only for the $i$-th machine in the process line.

According to the stages of higher and lower energy consumptions along $T_{M_{i}}$, the activation instants of each peripheral device should be selected taking into account both its operating constraints and its dependency on the activation sequence of machines $\boldsymbol{\Lambda}_{\mathbf{M}_{\mathbf{i}}}$. Thus, considering a fixed number of both ma- chines and peripheral devices in a process line, their activation sequences can be defined as

$$
\begin{aligned}
\boldsymbol{\Lambda}_{\mathbf{M}_{\mathbf{i}}}(k) & =\left\{u_{M_{i, 1}}(k), u_{M_{i, 2}}(k), \ldots, u_{M_{i, m}}(k)\right\}, \\
\boldsymbol{\Lambda}_{\mathbf{P}}(k) & =\left\{u_{P_{1}}(k), u_{P_{2}}(k), \ldots, u_{P_{n}}(k)\right\},
\end{aligned}
$$

where $k \in \mathbb{Z}_{\geq 0}$ is the discrete-time index and, $m=\left|\boldsymbol{\Lambda}_{\mathbf{M}_{\mathbf{i}}}\right|$ and $n=\left|\boldsymbol{\Lambda}_{\mathbf{P}}\right|$ are the total number of manufacturing devices of the $i$-th machine and the number of peripheral devices in the process line, respectively. Usually, the activation signals of both machine devices and peripheral devices are constrained to $u_{M_{i, l}}(k) \in\{0,1\}, l \in \mathcal{L} \triangleq\{1,2, \cdots, m\}$, and $u_{P_{j}}(k) \in\{0,1\}$, $j \in \mathcal{J} \triangleq\{1,2, \cdots, n\}$. However, for the cases in which the activation load of devices can be modulated, the activation signal will be constrained to $u_{M_{i, l}}, u_{P_{j}} \in \mathbb{Z}_{\geq 0}$.

For the case of machines, the execution times $T_{M_{i, l}}$ of the machine devices are usually fixed and their operation is constrained into $T_{M_{i}}$ (with $T_{M_{i}}$ as the upper bound). Usually, the machining sequences $\boldsymbol{\Lambda}_{\mathbf{M}_{\mathbf{i}}}(k)$ of each machine are known a priori from the process planning and scheduling designed for each piece. Thus, machining sequences are fixed and periodic over the time. In this regard, Assumption 1 is established for the operation of machines and their activation sequences $\boldsymbol{\Lambda}_{\mathbf{M}_{\mathbf{i}}}(k)$.

Assumption 1. The machining sequence $\mathbf{\Lambda}_{\mathbf{M}_{\mathbf{i}}}$ is given and hence its associated apparent power consumption, denoted by $\beta_{i} \triangleq \sum_{k=0}^{T} S_{M_{i}}\left(\boldsymbol{\Lambda}_{\mathbf{M}_{\mathbf{i}}}(k)\right)$, when a periodic behaviour is considered.

On the other hand, the power consumption of peripheral devices $S_{P_{j}}$ depends on the operational relationships between them and machines in the process line since they must guarantee resources required for the manufacturing processes. Thus, in order to select the suitable activation instants of the peripheral devices that allow minimising the global energy consumption $S$ of the process line, the dynamics of both energy consumption and the process performed by the peripheral devices, and operating constraints between them and machines should be taken into account for the design of control strategies. In this regard, the control problem consists of determining the optimal $\Lambda_{\mathbf{P}}$ that minimises the global $S$ along a fixed period $T$. Indeed, the control objective can be defined as the minimisation of the integral of the energy consumption signal, i.e.,

$$
J(k)=\sum_{k=1}^{T}\left[\sum_{i=1}^{b} S_{M_{i}}(k)+\sum_{j=1}^{n} S_{P_{j}}(k)\right] \Delta k,
$$

being $b$ the total number of machines in the process line and $\Delta k$ the sampling time. Then, to compute the global apparent power consumption $S(k)=\sum_{j=1}^{n} S_{P}(k)+\sum_{i=1}^{b} S_{M_{i}}(k)$, power consumption dynamical models for each machine

$$
\begin{aligned}
\xi_{M_{i}}(k+1) & =h_{1}\left(\xi_{M_{i}}(k),\left\{u_{M_{i, 1}}(k), u_{M_{i, 2}}(k), \cdots, u_{M_{i, l}}(k)\right\}\right) \\
S_{M_{i}}(k) & =h_{2}\left(\xi_{M_{i}}(k)\right),
\end{aligned}
$$

and each peripheral device are required, i.e., 


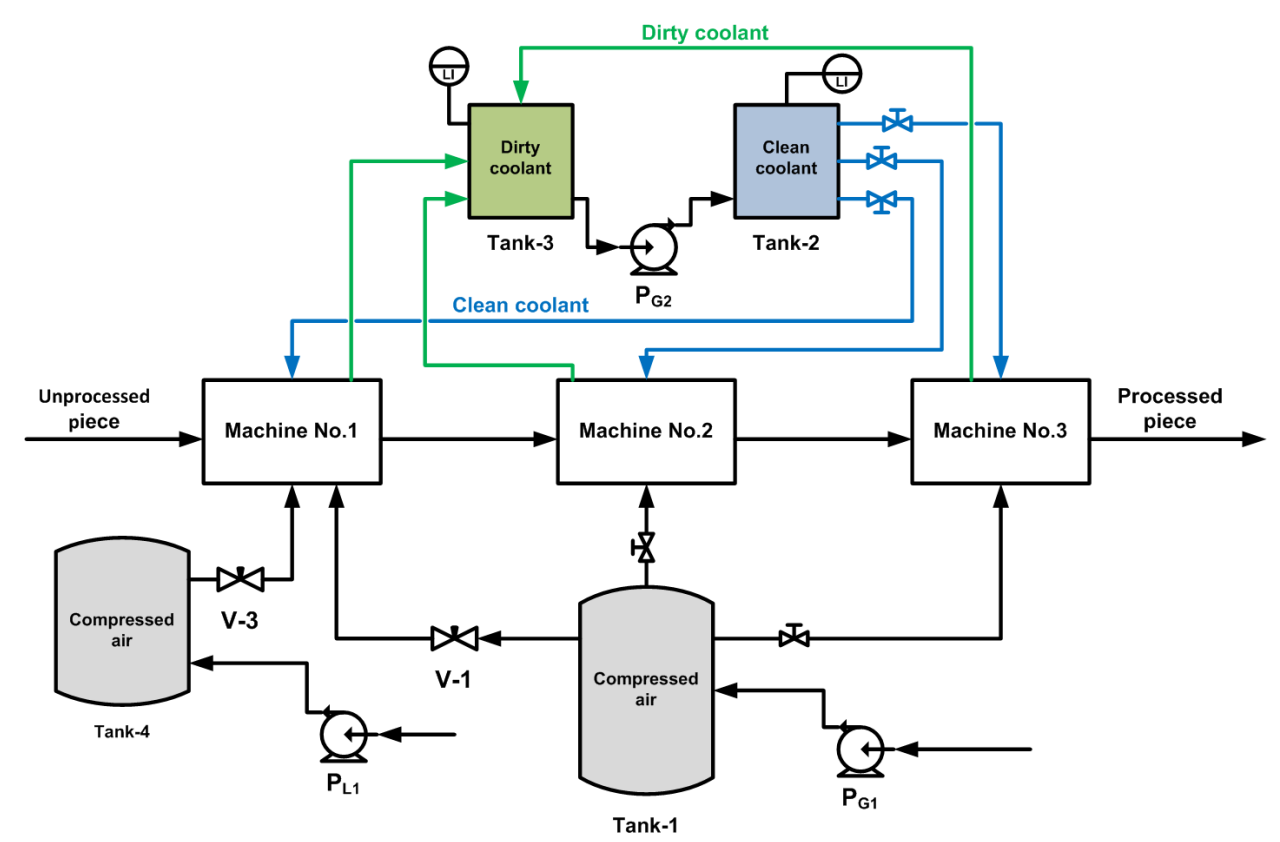

Figure 1: Three-stage serial process line with its corresponding peripheral systems.

$$
\begin{aligned}
\gamma_{P_{j}}(k+1) & =f_{1}\left(\gamma_{P_{j}}(k), u_{P_{j}}(k)\right), \\
S_{P_{j}}(k) & =f_{2}\left(\gamma_{P_{j}}(k)\right),
\end{aligned}
$$

where $\gamma_{P_{j}}(k) \in \mathbb{R}^{p}$ ans $\xi_{M_{i, l}}(k) \in \mathbb{R}^{r}$ are the system states of the energy consumption model related to $P_{j}$ and $M_{i, l}$, respectively. Besides, $f_{1}: \mathbb{R}^{p} \times\{0,1\}^{n} \mapsto \mathbb{R}^{p}$ and $f_{2}: \mathbb{R}^{p} \mapsto \mathbb{R}_{\geq 0}$ are the maps in function of both the current state and the activation signal of $P_{j}$, while $h_{1}: \mathbb{R}^{r} \times\{0,1\}^{m} \mapsto \mathbb{R}^{r}$ and $h_{2}: \mathbb{R}^{r} \mapsto \mathbb{R}_{\geq 0}$ are the maps for $M_{i, l}$. Moreover, mathematical expressions for the dynamics of processes performed by peripheral devices, i.e.,

$$
q_{P_{j}}(k+1)=g_{P_{j}}\left(q_{P_{j}}(k), u_{P_{j}}(k), \boldsymbol{\Lambda}_{\mathbf{M}_{\mathbf{i}}}\right),
$$

are also required to express the operational relationships between machines and peripheral devices. In (5), $q_{P_{j}}(k)$ corresponds to states related to the dynamics of peripheral devices $P_{j}$ with $g_{P_{j}}: \mathbb{R} \times\{0,1\}^{n+m} \mapsto \mathbb{R}_{\geq 0}$ the maps that consider the relationships between machines and peripheral devices.

\section{A Benchmark System}

According to Figure 1, a process line with three machines and three peripheral devices will be used as a case study for the performance of the control strategy to be proposed. In order to analyse a scenario of flexible manufacturing, two different production programs to process two different types of pieces will be considered. The difference between the production programs concerns to the first machine, for which the resource consumption at each instant $k$ will be higher when the second production program is executed. In this case, it is assumed that all machines in the process line have the same cycle time, i.e.,
$T_{M_{i}}=28 \mathrm{~s} \forall i=1,2,3$ and their machine sequences ${ }^{1} \boldsymbol{\Lambda}_{\mathbf{M}_{\mathbf{i}}}(k)$ are fixed and periodic over the time. The peripheral devices $P_{G_{1}}$ and $P_{G_{2}}$ are considered as global peripheral devices since they are shared among the three machines in the process line. In contrast, $P_{L_{1}}$ is a local device that only provides resource to $M_{1}$. Thus, for the first production program, the global devices $P_{G_{1}}$ and $P_{G_{2}}$ are able to supply the resources demand of machines. However, when the second production program is executed, an extra peripheral device $\left(P_{L_{1}}\right)$ is required since the device $P_{G_{1}}$ is not able to supply the new demand of compressed air required by machines activated in the second program.

Both $P_{G_{1}}$ and $P_{L_{1}}$ are associated to the supply system of compressed air, which will be used for clamping pieces during the whole machining sequence. Besides, it is assumed that both $P_{G_{1}}$ and $P_{L_{1}}$ have a nominal energy consumption whenever the device is turned on. It should be noted that the local peripheral device $P_{L_{1}}$ works only for $M_{1}$ and its operation depends on the production program executed in the process line. Thus, in order to consider a flexible manufacturing scenario, it is assumed that $P_{L_{1}}$ will be inactivated during the first production program, and will be activated when the second production program is executed since the resource consumption of $M_{1}$ during the second program is significantly higher than its consumption in the previous production program. For the case in which the second production program will be executed because a different part should be processed in the process line, new decision variables to determine the optimal flow to be taken from each device are required. Thus, according to Figure 1 the aperture of the valves $v_{1}$ and $v_{3}$ should also be optimised according to the new sequences of resources consumption of machines for the new production program. It should be noted that the other

\footnotetext{
${ }^{1}$ It corresponds to the sequence of processes performed by the machining devices of a machine to process a piece.
} 
valves in the process line are not directly manipulated since it is assumed that they are opened/closed when required, and they can provide the flows demanded by the machines. Thus, without loss of generality, in this case, only the valves involved in coupled dynamics will be addressed in the extended operation range.

On the other hand, $P_{G_{2}}$ is related to a coolant supply system for the machining operations at each machine, and its activation could be modulated to different energy consumption levels. According to this, both the activation instant and the activation level for $P_{G_{2}}$ should be optimised according to the resources consumption from machines. It is worth noting that the consumption of resources from machines depends on $\boldsymbol{\Lambda}_{\mathbf{M}_{\mathrm{i}}}$ and the production program, and although in this paper the machining sequences are not presented in detail since they are assumed to be fixed and constant over the time, in Figure 2, the consumption profile of compressed air and coolant from machines is presented. Based on Figure 2, it is possible to observe that the unique difference between the production programs regards the machine $M_{1}$, in which the resource consumption is duplicated, as shown in this figure with a red line.

\subsection{Air-supply system}

One of the functions of pneumatic systems is to supply air of pre-defined conditions of pressure to clamp/unclamp pieces during manufacturing operations. Considering the supply system shown in Figure 1, a pump provides to each air stream the necessary energy to achieve the desired conditions of pressure, and then, this stream is transported towards the devices that effectively use it. Based on this fact, the dynamics associated to $P_{G_{1}}$ (and $P_{L_{1}}$ ) correspond to the dynamics for the total mass exchange $M_{T_{1}}\left(M_{T_{4}}\right)$ and pressure $P_{T_{1}}$ (and $P_{T_{4}}$ ) inside a storage tank $T_{1}$ (and $T_{4}$ ), which are expressed in the discrete-time version below. It is worth noting that the equations are presented with respect to the tank 1 , and a similar set of equations can be written for the tank 4 .

$$
\begin{aligned}
M_{T_{1}}(k+1) & =M_{T_{1}}(k)+\tau_{s} \sigma(k), \\
\sigma(k) & =m_{\text {in }} u_{G_{1}}(k)-m_{G, 1_{M_{1}}}-\sum_{i=2}^{b} m_{\text {air }, M_{i}}(k), \\
P_{T_{1}}(k) & =\frac{M_{T_{1}}(k) R T}{V_{T_{1}} W_{\text {air }}},
\end{aligned}
$$

being $\tau_{s}$ the sampling time, $m_{a i r, M_{i}}$ the air consumption from machine $M_{i}, m_{i n}$ the air flow pumped by $P_{G_{1}}$ (and $P_{L_{1}}$ ) towards the tank $T_{1}$ (and $T_{4}$ ), and, $R, T, V_{T_{1}}$ and $W_{\text {air }}$ the gas constant, air temperature, volume of $T_{1}$ (and $T_{4}$ ), and the molecular weight, respectively. In addition, the pressure $P_{T_{1}}$ (and $P_{T_{4}}$ ) must satisfy $\underline{P}_{T_{1}} \leq P_{T_{1}}(k) \leq \bar{P}_{T_{1}}$ (and $\left.\underline{P}_{T_{4}} \leq P_{T_{4}}(k) \leq \bar{P}_{T_{4}}\right)$, with $\underline{P}_{T_{1}}$ and $\leq$ $\bar{P}_{T_{1}}$ the lower and upper bounds for $P_{1}$, respectively. Moreover, since $P_{L_{1}}$ is a local device of $M_{1}$, the second term in the righthand side in (6b) does not exist and the only output of $T_{4}$ is $m_{L, 1_{M_{1}}}$.

It should be noted that for the case in which both $P_{L_{1}}$ and $P_{G_{1}}$ provide compressed air to $M_{1}$ at the same time, the sum of
$m_{G, 1_{M_{1}}}$ and $m_{L, 1_{M_{1}}}$ should be equal the flow required by $M_{1}$ at each instant $k$, namely $m_{\text {air, } M_{1}}(k)$. In this regard, two valves with modulated aperture are considered into the process scheme to determine how much flow of compressed air could be provided from $P_{G_{1}}$ and $P_{L_{1}}$. The domain for variables $v_{1}$ and $v_{3}$ should be defined taking into account the maximum flow that can supply each device when the valves are $100 \%$ open as well as the admissible values into its domain to guarantee a feasible solution. Then, the energy consumption concerning valves is computed according to:

$$
\begin{aligned}
& S_{v_{1}}(k)=\alpha_{v_{1}} A p_{v_{1}}(k), \\
& S_{v_{3}}(k)=\alpha_{v_{3}} A p_{v_{3}}(k),
\end{aligned}
$$

being $\alpha_{v_{1}}$ and $\alpha_{v_{3}}$ the constant energy consumption of valves $v_{1}$ and $v_{3}$, respectively. Besides, the flows of compressed air provided by $P_{G_{1}}$ and $P_{L_{1}}$, according to the values of both $A p_{v_{1}}$ and $A p_{v_{3}}$, can be compute according to the following equations:

$$
\begin{aligned}
& m_{G, 1_{M_{1}}}(k)=\varepsilon_{M_{1}} A p_{v_{1}}(k), \\
& m_{L, 1_{M_{1}}}(k)=\varepsilon_{M_{3}} A p_{v_{3}}(k),
\end{aligned}
$$

with $\varepsilon_{M_{1}}$ and $\varepsilon_{M_{3}}$ the maximum flows provided by devices $P_{G_{1}}$ and $P_{L_{1}}$, respectively.

\subsection{Coolant-supply system}

The supply of coolant is a critical feature of the manufacturing processes. If the coolant is not supplied in either sufficient quantity or at suitable time instants, the manufacturing processes are not functioning at the correct regime, and the desired properties of the piece will not be reached. In many cases, coolant-supply systems are designed with re-circulation, filtering and re-use of coolant, as shown in Figure 1. According to this figure, a constant flow of coolant $m_{c}$ is pumped from $T_{3}$ towards a clean-coolant tank $T_{2}$ passing through a filter in which the fine particles are separated. Next, the coolant flows required by the machines are pumped from $T_{2}$. After manufacturing processes in the machine, the dirty coolant is collected and delivered to the tank $T_{3}$ where gross chip particles are separated by gravity, and the process is repeated. In this case, the pump $P_{G, 2}$ corresponds to the peripheral device of interest, and both the activation instant and the suitable flow of coolant to satisfy the operating constraints must be selected. Therefore, $u_{G_{2}} \in \mathbb{V}_{2} \subset \mathbb{Z}$ such that $\mathbb{V}_{2} \triangleq\{0,100,120,140\}$. The dynamics for level changes in both tanks are the following:

$$
\begin{gathered}
L_{2}(k+1)=L_{2}(k)+\tau_{s} \gamma(k)\left(\frac{1}{\rho_{c} A_{T_{2}}}\right), \\
\gamma(k)=m_{c}(k)-\sum_{i=1}^{b} m_{c c l, M_{i}}(k), \\
L_{3}(k+1)=L_{3}(k)+\tau_{s} \theta(k)\left(\frac{1}{\rho_{c} A_{T_{3}}}\right), \\
\theta(k)=\sum_{i=1}^{b} m_{d c l, M_{i}}(k)-m_{c}(k),
\end{gathered}
$$



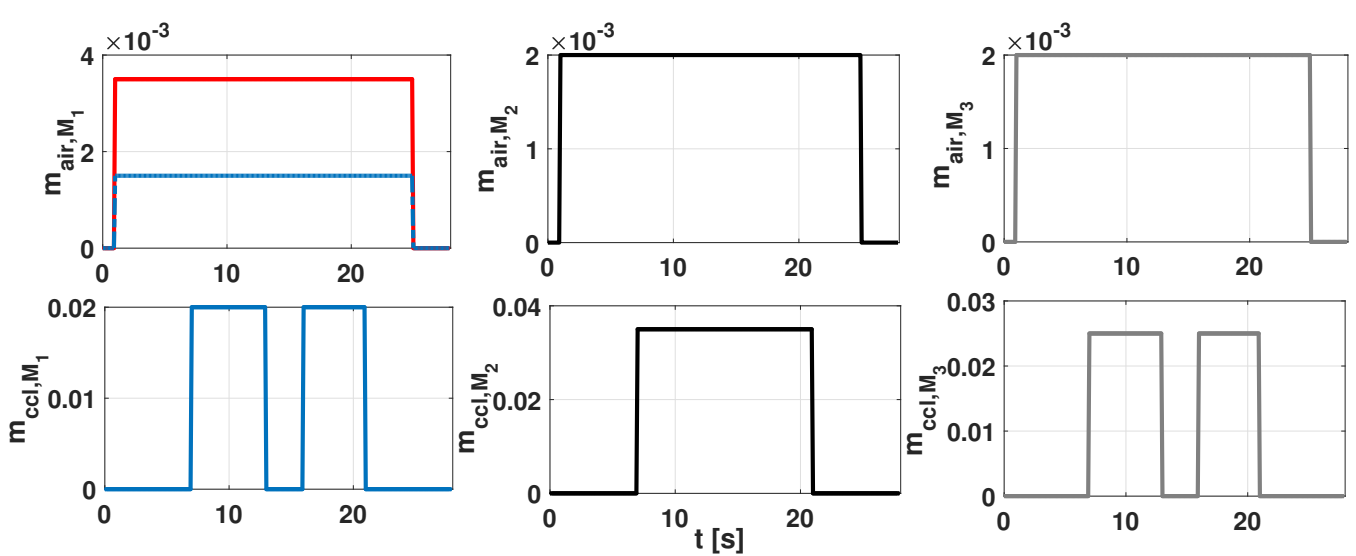

Figure 2: Sequences for the flows of compressed air (top) and coolant (bottom) in $\left[\frac{\mathrm{kg}}{\mathrm{s}}\right]$ consumed from machines in the process line along $T_{M_{i}}$ according to their machining sequences. For the case of $M_{1}$, the blue line refers to the air consumption when the first production program is executed, and the red line corresponds to the consumption when the second production program is activated.

Table 1: Physical dimensions and parameters for the supply systems of compressed air and coolant.

\begin{tabular}{cccc}
\hline Parameter & Value & Parameter & Value \\
\hline$V_{T_{1}}$ & $0.015 \mathrm{~m}^{3}$ & $V_{T_{4}}$ & $0.01 \mathrm{~m}^{3}$ \\
$A_{T_{2}}$ & $0.015 \mathrm{~m}^{2}$ & $A_{T_{3}}$ & $0.015 \mathrm{~m}^{2}$ \\
$T_{\text {air }}$ & $25^{\circ} \mathrm{C}$ & $R$ & $8.1314 \frac{\mathrm{J}}{\mathrm{Kmol}}$ \\
$W_{\text {air }}$ & $28.966 \frac{\mathrm{g}}{\mathrm{mol}}$ & $\Delta P_{\text {filter }}$ & $10000 \mathrm{~Pa}$ \\
$P_{\text {atm }}$ & $101325 \mathrm{~Pa}$ & $\eta$ & 0.95 \\
$\rho_{c}$ & $1042.5 \frac{\mathrm{kg}}{\mathrm{m}}$ & $h_{1 \rightarrow 2}$ & $0.12 \frac{\mathrm{m}^{2}}{\mathrm{~s}^{2}}$ \\
$m_{\text {in, } 1}$ & $0.006 \frac{\mathrm{kg}}{\mathrm{s}}$ & $m_{\text {in, } 3}$ & $0.004 \frac{\mathrm{kg}}{\mathrm{s}}$ \\
$\alpha_{v_{1}}$ & $2.5 \mathrm{VA}$ & $\alpha_{v_{3}}$ & $2.5 \mathrm{VA}$ \\
$\varepsilon_{M_{1}}$ & $1.5 \times 10^{-5}$ & $\varepsilon_{M_{3}}$ & $3.0 \times 10^{-5}$ \\
$\underline{P}_{T_{1}}$ & $300 \mathrm{kPa}$ & $\bar{P}_{T_{1}}$ & $750 \mathrm{kPa}$ \\
$\underline{P}_{T_{4}}$ & $300 \mathrm{kPa}$ & $\bar{P}_{T_{4}}$ & $750 \mathrm{kPa}$ \\
$\underline{L}_{T_{2}}$ & $0.3 \mathrm{~m}$ & $\bar{L}_{T_{2}}$ & $0.6 \mathrm{~m}$ \\
$\underline{L}_{T_{3}}$ & $0.4 \mathrm{~m}$ & $\bar{L}_{T_{3}}$ & $0.7 \mathrm{~m}$ \\
\hline
\end{tabular}

with $m_{c}$ given by

$$
m_{c}(k)=\frac{\eta \rho_{c} W(k)}{P_{\text {in }}(k)+\rho_{c} h_{f_{1 \rightarrow 2}}(k)-P_{\text {out }}(k)},
$$

and being $m_{c c l_{M_{i}}}$ the coolant flow required by the $i$-th machine, and $m_{d c l_{M_{i}}}$ the flow of the dirty coolant recovered. In addition, $P_{\text {in }}$ and $P_{\text {out }}$ correspond to the input and output pressure in the pipe system that transport the coolant from $T_{3}$ towards $T_{2}$, while, $\rho_{c}, \eta, W$ and $h_{f_{1 \rightarrow 2}}$ are the coolant density, efficiency of the pump, the work supply to the pump $P_{G, 2}$ and the energy losses by friction, respectively. In this regard, the activation instants and the coolant flow could be determined to satisfy the operating levels at each tank. Thus, $W$ is directly related to the activation signal of the pump $P_{G, 2}$. In Table 1 , the physical dimensions of the coolant system and the two air supply systems are presented.

\subsection{Energy consumption}

Different approaches have been proposed for modelling and designing control strategies in manufacturing systems. Among others, the Markov chains (MC) and Petri Nets (PN) can be highlighted given their great application at both process line and plant levels [12]. Despite the important impact in applications of both MC and PN for the manufacturing systems, they have been focused on modelling the production states of the machine rather than to model the energy consumption behaviour of such systems. Thus, since the complexity to establish the different machine states, transitions between them, and their connections, data-driven models, such as those obtained from the Subspace Identification (SI) methods, have gained attention into the manufacturing industry to model the specific energy consumption.

Thereby, for determining the power consumption models of both manufacturing and peripheral devices, SI methods are addressed in this section. The SI methods allow identifying the matrices of a state-space realisation of linear time-invariant (LTI) systems based on input-output data. These algorithms are useful since state-space realisations are convenient for estimation, control and prediction tasks. These methods start from the idea that a set of measurements of $b$ input signals $(b \geq 1)$ and $p$ output signals $(p \geq 1)$ are related through a $N$-order state-space (unknown) realisation as follows:

$$
\begin{aligned}
\mathbf{x}(k+1) & =A \mathbf{x}(k)+B \mathbf{u}(k)+\mathbf{w}(k), \\
\mathbf{y}(k) & =C \mathbf{x}(k)+D \mathbf{u}(k)+\mathbf{v}(k),
\end{aligned}
$$

with $\mathbf{x} \in \mathbb{R}^{\ell}, \mathbf{u} \in \mathbb{R}^{b}$, and $\mathbf{y} \in \mathbb{R}^{f}$ are the state, input and output vectors, while $\mathbf{w} \in \mathbb{R}^{\ell}$ and $\mathbf{v} \in \mathbb{R}^{p}$ are the state noise and output measurement noise, respectively. In a deterministic case, in which $\mathbf{w}$ and $\mathbf{v}$ are neglected, the SI problem consists of estimating the system order $N$ and the system matrices $A \in \mathbb{R}^{\ell \times \ell}, B \in \mathbb{R}^{\ell \times b}, C \in \mathbb{R}^{p \times \ell}$, and $D \in \mathbb{R}^{p \times b}[11]$.

In order to obtain a state-space representation of energy consumption models as shown in (4), this paper focuses on the $\mathrm{Nu}$ merical algorithms For Subspace IDentification (N4SID) [16] 
recommended by their versatility and availability in terms of numerical methods. The N4SID algorithms first estimate the state $\hat{x}$ from the projection of input-output data, and then, systems matrices are determined based on the estimated state sequence. A detailed review about the different SI algorithms and their implementation can be found in $[13,16]$.

Thereby, to get suitable data sets for the modelling process, different activation sequences for both $\boldsymbol{\Lambda}_{\mathbf{M}_{\mathbf{i}}}$ of each machine along $T_{M_{i}}$ and $\boldsymbol{\Lambda}_{\mathbf{P}}$ should be tested to get information about their resulting energy consumption. In our case study, three different machine sequences $\boldsymbol{\Lambda}_{\mathbf{M}_{\mathbf{i}}}$ (each one per machine in the process line) were designed emulating the different processing stages performed by a real machine tool. Based on these sequences, data for the energy consumption along $T_{M_{i}}$ was collected from a test bench built to emulate the energy consumption of a machine tool $S_{M_{i}}$. Then, using $\boldsymbol{\Lambda}_{\mathbf{M}_{\mathbf{i}}}$ and $S_{M_{i}}$ as input and output data, respectively, the energy consumption model for a machine cycle is obtained. Based on this model, the energy consumption profile of the machine when a piece is processed is defined. It is worth noting that this profile will be fixed and periodic along the time. Next, for the case of peripheral devices, since they are not constrained to a machine cycle, different sequences of $\boldsymbol{\Lambda}_{\mathbf{P}}$ considering different activation times were tested to obtain a rich range of the corresponding outputs $S_{P_{j}}$. In the same way, $\boldsymbol{\Lambda}_{\mathbf{P}}$ and $S_{P_{j}}$ for each device were used as input-output data set to identify their energy consumption models.

\section{Control Mode Switching Strategy based on MPC}

Based on the stages of both high and low energy consumption into the corresponding profile for a machine, peripheral devices must be correctly managed in order to minimise the total energy consumption and to avoid also (whenever possible) their simultaneous activation. Thus, to design a control strategy such that it does not affect the productivity of the process line, Assumption 1 was stated. Based on this, $\boldsymbol{\Lambda}_{\boldsymbol{M}_{i}}$, which represent the activation sequence of machining devices, is considered as fixed and periodic over the time, assuming they are already optimised with respect to the energy consumption. This latter fact implies that the time $T_{M_{i}}$ to process a piece at each $M_{i}$ remains the same and, therefore, the process line has the capacity to handle the same number of pieces as when the control/supervision strategy is not implemented. Thus, since the operation of machines cannot be modified, only peripheral devices will be managed to minimise the total energy consumption (machines and peripheral devices) taking into account the operational relationships among machines and peripheral devices.

In this regard, an optimisation-based control strategy that allows minimising the global energy consumption of a process line is proposed taking into account its dynamics, the global energy consumption $S$ and, the operational relationships between machines and peripheral devices in the process line. Besides, the control strategy should be able to respond to changes in production programs and to adapt its operation according to the new operating constraints, relationships among the process-line components, and the new energy consumption profiles of ma- chines. Therefore, the proposed control strategy is designed considering two different control modes:

- Predictive control mode: This mode is based on the design of a prediction-based controller according to the Model Predictive Control (MPC) approach to determine the optimal activation sequence of peripheral devices in the process line $\boldsymbol{\Lambda}_{\mathbf{P}}^{*}$ at the current production program. For the case of the second production program, the optimal sequences for the aperture of valves $\boldsymbol{\Lambda}_{\mathbf{V}}^{*}$ should also be determined.

- Autonomous control mode: The second control mode will be implemented only if the peripheral devices exhibit a periodic behaviour after a time period of running the predictive control mode. Then, if some peripheral device has a periodic behaviour, its periodicity should be detected and the activation sequence previously optimised in the first control mode (i.e., $\boldsymbol{\Lambda}_{\mathbf{P}}^{*}$ and $\boldsymbol{\Lambda}_{\mathbf{V}}^{*}$ ) will be sent to the system. That means, every time the energy response of a peripheral device is detected as periodic, it will be removed from the optimisation problem underlying the controller design in the former control mode with the aim to decrease the computational burden.

The predictive control mode is based on both energy consumption models and operating constraints of peripheral devices embedded in an optimisation problem behind the design of the controller. The general idea of this mode is to anticipate either activation or deactivation of peripheral devices that allow minimising the global energy consumption of the process line taking into account their dynamics, the global energy consumption $S$ and the operational relationships with the machines in the process line. Then, considering a prediction horizon $H_{p}=T$ (with $T=\max \left(T_{M_{i}}\right)$ ), the decision of switching on or off a device $j$ depends on the current value of the energy consumption of machines $S_{M_{i}}(k)$, i.e., although the manufacturing sequences for machines in the process line are already given and hence its energy consumption profile along $T_{M_{i}}$, its real consumption values discriminated along the time are important for making decisions regarding real-time peripheral devices management.

According to the control objective defined in (2), the sequences $^{2}$ for $J, \Lambda_{\mathbf{P}}$ and $\Lambda_{\mathbf{V}}$ along $H_{p}$ are defined as

$$
\begin{aligned}
\mathbf{J}(k) & \triangleq\left\{J(k \mid k), \ldots J\left(k+H_{p}-1 \mid k\right)\right\}, \\
\boldsymbol{\Gamma}(k) & \triangleq\left\{\boldsymbol{\Lambda}_{\mathbf{P}}(k \mid k), \ldots, \boldsymbol{\Lambda}_{\mathbf{P}}\left(k+H_{p}-1 \mid k\right)\right\}, \\
\boldsymbol{\Pi}(k) & \triangleq\left\{\boldsymbol{\Lambda}_{\mathbf{V}}(k \mid k), \ldots, \boldsymbol{\Lambda}_{\mathbf{V}}\left(k+H_{p}-1 \mid k\right)\right\},
\end{aligned}
$$

with $\boldsymbol{\Lambda}_{\mathbf{V}}(k)=\left\{v_{1}(k), v_{3}(k)\right\}, \mathbf{J}(k) \in \mathbb{R}^{H_{p}}, \boldsymbol{\Gamma}(k) \in\{0,1\} \times \mathbb{V}_{2}^{n H_{p}}$, and $\Pi(k) \in\{0,100\}^{n H_{p}}$.

Subsequently, the design of the proposed prediction-based controller is based on the following finite-time open-loop optimisation problem:

\footnotetext{
${ }^{2}$ Here, $z(k+i \mid k)$ denotes the prediction of the variable $z$ at time instant $k+i$ performed at $k$. The index $i$ will cover the finite prediction window of length
} $H_{p}$ 


$$
\min _{\Gamma(\mathbf{k}), \Pi(\mathbf{k})} \mathbf{J}(k)
$$

subject to

$$
\begin{aligned}
\gamma_{G_{1}}(k+i+1) & =f_{1}\left(\gamma_{G_{1}}(k+i), u_{G_{1}}(k+i)\right), \\
S_{G_{1}}(k+i) & =f_{2}\left(\gamma_{G_{l}}(k+i)\right), \\
\gamma_{G_{2}}(k+i+1) & =f_{3}\left(\gamma_{G_{2}}(k+i), u_{G_{2}}(k+i)\right), \\
S_{G_{2}}(k+i) & =f_{4}\left(\gamma_{G_{2}}(k+i)\right), \\
\gamma_{L_{1}}(k+i+1) & =f_{5}\left(\gamma_{L_{1}}(k+i), u_{L_{1}}(k+i)\right), \\
S_{L_{1}}(k+i) & =f_{6}\left(\gamma_{L_{l}}(k+i)\right), \\
q_{G_{1}}(k+i+1) & =g_{G_{1}}\left(q_{G_{1}}(k+i), u_{G_{l}}(k+i), \boldsymbol{\Lambda}_{\mathbf{M}_{\mathbf{i}}}\right), \\
q_{G_{2}}(k+i+1) & =g_{G_{2}}\left(q_{G_{2}}(k+i), u_{G_{2}}(k+i), \boldsymbol{\Lambda}_{\mathbf{M}_{\mathbf{i}}}\right), \\
q_{L_{1}}(k+i+1) & =g_{L_{1}}\left(q_{L_{1}}(k+i), u_{L_{l}}(k+i), \boldsymbol{\Lambda}_{\mathbf{M}_{1}}\right), \\
u_{G_{1}}(k+i) & \in\{0,1\}, \\
u_{G_{2}}(k+i) & \in \mathbb{V}_{2}, \\
u_{L_{1}}(k+i) & \in\{0,1\}, \\
v_{1}(k+i) & \in\{0,100\}, \\
v_{3}(k+i) & \in\{0,100\}, \\
q_{G_{1}}(k+i) & \in\left[\bar{q}_{G_{1}}, \underline{q}_{G_{1}}\right], \\
q_{G_{2}}(k+i) & \in\left[\bar{q}_{G_{2}}, \underline{q}_{G_{2}}\right], \\
q_{L_{1}}(k+i) & \in\left[\bar{q}_{L_{1}}, \underline{q}_{L_{1}}\right],
\end{aligned}
$$

and the following logical constraints to avoid the high switching frequency of system actuators:

$$
\Delta u_{j}(k+i) \neq 0 \Longleftrightarrow u_{j}(k+i+l)=u_{j}(k+i), \quad \forall l=1, \cdots, 4,
$$

for $i \in\left\{0,1,2, . ., H_{p}-1\right\}$, and being $\gamma_{G_{1}}, \gamma_{G_{2}}$ and $\gamma_{L_{1}}$ the states of energy consumption models for $P_{G_{1}}, P_{G_{2}}$ and $P_{L_{1}}$, respectively, $q_{G_{1}}, q_{G_{2}}$ and $q_{L_{1}}$ the states of peripheral devices, and $\Delta u_{j}(k)=\left|u_{j}(k)-u_{j}(k-1)\right|$ the activation/deactivation indicator of the $j$-th peripheral device. The $q_{j}$-dynamics in (13) correspond to the process dynamics for the compressed air supply system and the coolant supply system previously described in Section 2 for the peripheral devices in the process line, i.e., $P_{G_{1}}, P_{L_{1}}$ and $P_{G_{2}}$. It should be noted that when the first production program is running, the device $P_{L_{1}}$ is deactivated, and therefore, its activation sequence is restricted to be null along the time.

If it is assumed that the problem (13) is feasible, i.e., $\boldsymbol{\Gamma}(k) \neq$ $\emptyset, \Pi(k) \neq \emptyset$, and thus it exists an optimal solution for the activation sequences of peripheral devices and valves defined by

$$
\begin{aligned}
\boldsymbol{\Gamma}^{*}(k) \triangleq\left\{\boldsymbol{\Lambda}_{\mathbf{P}}{ }^{*}(k \mid k), \ldots, \boldsymbol{\Lambda}_{\mathbf{P}}{ }^{*}\left(k+H_{p}-1 \mid k\right)\right\}, \\
\boldsymbol{\Pi}^{*}(k) \triangleq\left\{\boldsymbol{\Lambda}_{\mathbf{V}}{ }^{*}(k \mid k), \ldots, \boldsymbol{\Lambda}_{\mathbf{V}}{ }^{*}\left(k+H_{p}-1 \mid k\right)\right\} .
\end{aligned}
$$

According to the receding horizon philosophy [10, 14], $\boldsymbol{\Lambda}_{\mathbf{P}}{ }^{*}(k \mid k)$ and $\boldsymbol{\Lambda}_{\mathbf{V}}{ }^{*}(k \mid k)$ are sent to the process line discarding the rest of the optimal sequences from $(k+1) \mid k$ to $\left(k+H_{p}-1\right) \mid k$, while the whole process is repeated at the next instant $k \in \mathbb{Z}_{\geq 0}$ after the updates on the measurements/estimations of the state information from the plant for both the energy consumption models and process dynamics considered in (13). In accordance with Section 3.3, data-driven models have been selected to represent the energy consumption models of peripheral devices since the physical-based models require the full knowledge of several physical dynamics and parameters, which are often hard to represent, compute or estimate. Thus, linear statespace representations based on the real input-output data sets were obtained by using system identification algorithms.

On the other hand, the second control mode (or the autonomous mode) will be activated when a periodic behaviour in the optimal activation/deactivation signal of each peripheral device is identified. The decision on the activation of this mode is take globally and then at the level of each device, the periodic pattern is monitored according to the dynamics of their associated processes. Once the periodic behaviour is identified at the level of a particular device, it will be removed of the optimisation problem (13) to reduce both the number of decision variables and the computational burden. Besides, the periodic sequence detected is sent to the corresponding plant input at each time instant $k$, while the optimisation for the other peripheral devices keeps taking also into account the energy consumption resulting from the devices that now entered in a periodic behaviour. Finally, if the periodicity is detected for all peripheral devices in the process line, the control commutes to a completely autonomous mode in which there is no optimisation in real time. Thus, the optimal and periodic sequences for the activation/deactivation of peripheral devices are fixed and sent to the plant and the control unit entering a monitoring phase where the performance indices are checked to verify the functioning according to the predefined pattern. In the case a mismatch is detected, a change of mode is triggered.

When the system is on autonomous mode, the computational burden is reduced since no online optimisation is required. However, in this mode it is not possible to manage any disturbance or change that might affect the operation of the peripheral devices and the machines in the process line. Therefore, the protocol for commuting from the autonomous mode to the predictive/optimisation mode is required. Since in the autonomous mode per se is not able to predict and find the activation/deactivation sequence of peripheral devices, a switching protocol is implemented while the autonomous mode is running, in which a prediction of the process is performed considering both the current measurements of the plant and the predefined periodic control sequences. Thus, this protocol aims to verify that the system constraints will be satisfied in the future according to the current state of the system. In this regard, any change or disturbance appreciated in the measurements from the plant could produce an abnormal behaviour in the future if the same activation sequence is kept and sent to the plant. If it happens, the monitoring and commutation protocol should detect these abnormalities and should indicate that, at the current system conditions, the system state will be out of its feasible domain and, therefore the control sequence 
should be recomputed to enforce feasibility of operation and optimise the performance. Practically, when abnormalities are detected, the controller switch to the predictive (or first control) mode with the aim to determine on-line the new optimal activation/deactivation signals of peripheral devices at the current state of the process line.

To clarify the proposed dual control strategy, the control scheme proposed for the implementation of the control strategy in real time is shown in Figure 3. In the next sections, both the strategy for the detection of the periodicity of peripheral devices and the commutation protocol will be explained in detail.

\subsection{Periodicity Detection}

To determine the periodicity pattern within the operation of peripheral devices and to commute to the autonomous mode, we propose Algorithm 1 based on signal processing tools such as autocorrelation. Thus, after a time period $N_{u}$ of operating under the first control mode, the activation/deactivation sequence of each peripheral device that has been sent to the system $\mathbf{u}_{j}$ is used to determine its periodicity. At time instant $k \geq N_{u}$, a preprocessing of the activation/deactivation signal $\mathbf{u}_{j}$ is performed to remove its mean value and capture the more relevant changes of the signal $\left(\hat{\mathbf{u}}_{j}\right)$. It should be noted that this procedure is more important for those peripheral devices in which the activation load could be modulated since their periodicity should be detected taking into account both the activation instant and the level of activation. Thus, removing the mean value, the small changes associated with the load modulation gain relevance and the periodicity could be detected easier using autocorrelation. It is worth noting that the mean value is removed only for the periodicity-detection procedure. Once periodicity is found, the sequence to be sent to the process line will be taken from the original one without removing the mean value.

It is worth noting that, in this case, autocorrelation was selected since it allows identifying repetitive patterns in a signal such as its periodicity. Thus, autocorrelation is a measurement of the correlation/similarity between a signal and a delayed version of itself. In this regard, correlation coefficients are calculated considering different values of signal delays with respect to itself, and if the signal is periodic, it will be perfectly correlated with a version of itself if the time delay is an integer number of periods [6]. In this regard, given a discrete-time signal $y(k)$, the discrete autocorrelation $R_{y y}$ at lag $\tau_{y}$ is defined by

$$
R_{y y}\left(\tau_{y}\right)=\sum_{n \in \mathbb{Z}} y(k) y\left(k-\tau_{y}\right),
$$

where $R_{y y}$ is the correlation coefficient at the lag $\tau_{y}$. Thus, high values of $R_{y y}$ correspond to lags over which the the signal presents similar patterns. Then, in order to detect the periodicity, the autocorrelation of the signal is computed for all possibles lags and, the values in which it is higher are identified as possible periods of the signal.

Once the signal is pre-processing, the autocorrelation $\mathbf{u x}_{\mathbf{j}}$ of the pre-processed signal of $\hat{\mathbf{u}}_{j}$ is computed and the vectors of both correlation coefficients and lags are determined. Due to the

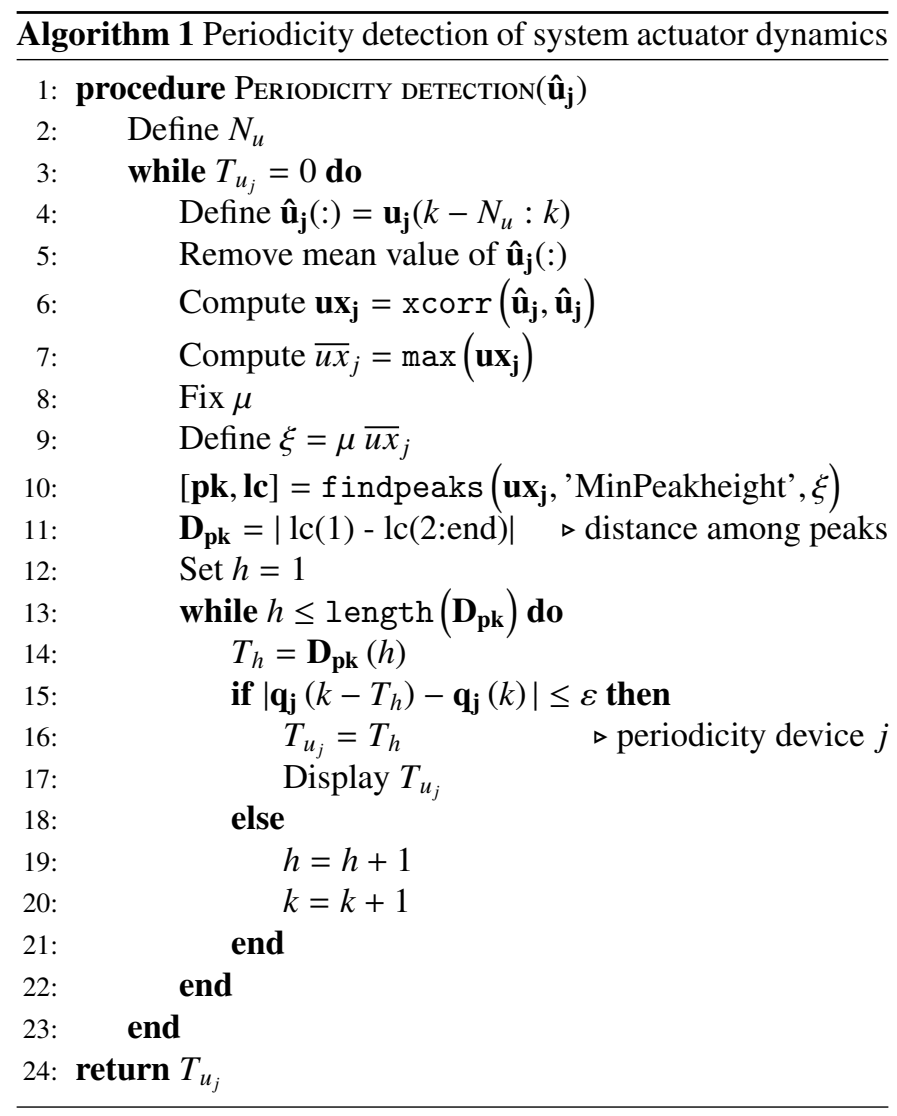

nature of the activation/deactivation signal (step signal), some patterns that do not correspond to the real period of the signal could be also identified and have significant values of $\mathbf{u x}_{\mathbf{j}}$. Therefore, the periodicity condition should be verified for the different lags obtained from autocorrelation. In this regard, the maximum value of $\overline{u x}_{j}=\mathbf{u x}_{\mathbf{j}}$ is determined, and then, only the peaks $\mathbf{p k}$ in $\mathbf{u x}_{\mathbf{j}}$ above the $\mu$ percentage of $\overline{u x}_{j}$ are considered to check the periodicity constraint, i.e.,

$$
\left|x(k)-x\left(k+p T_{x}\right)\right| \leq \varepsilon,
$$

$\forall p \in \mathbb{Z}$, being $T_{x}$ the period of the signal and $\varepsilon$ the tolerance value.

According to peaks pk and the corresponding values of lags lc, the distance between the first lag and the rest of the lags in lc is calculated. Then, the periodicity condition is verified for all distance previously computed, which represent the different alternatives to be chosen as the period of the signal. To this end, one of the signals for the dynamics of the processes associated to the operation of the peripheral device is considered. Thus, the period of the peripheral device $T_{u_{j}}$ will be defined equal to the distance in $\mathbf{D}_{\mathbf{p k}}$ for which the periodicity condition is satisfied. Afterwards, this device is removed from the optimisation problem considered in the first control mode. Finally, if the periodicity is found for all devices in the process line, then, the control system can commute to the autonomous control mode. It should be noted that if more than one value in $\mathbf{D}_{\mathbf{p k}}$ satisfies the periodicity condition, $T_{u_{j}}$ will be chosen according to the longer period to ensure the selection of the real period and not 


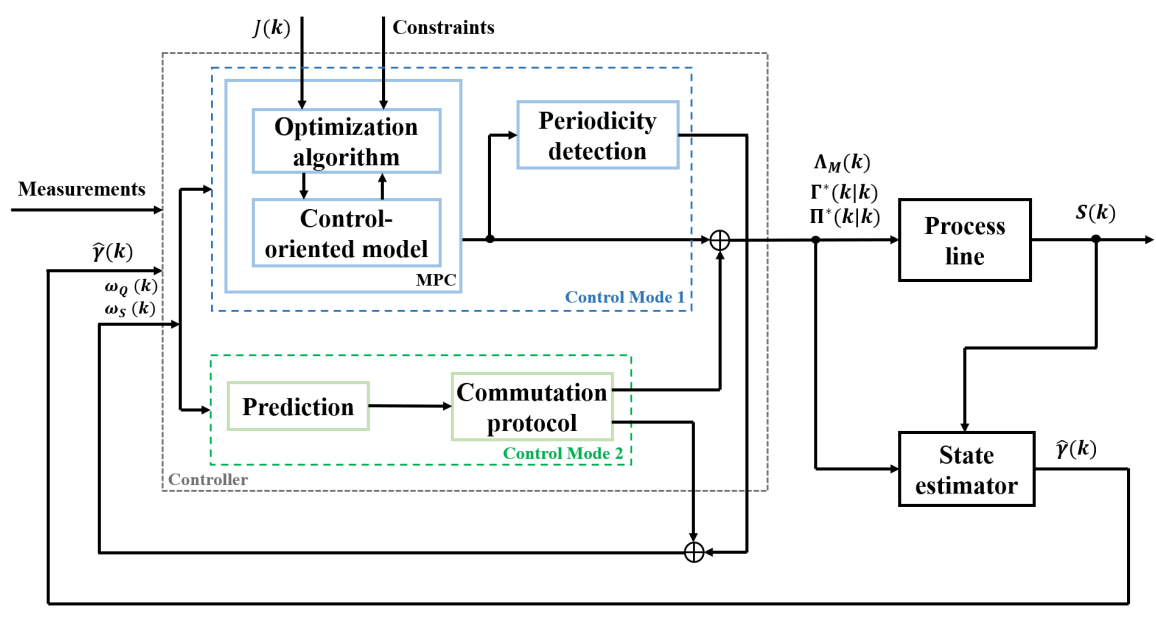

Figure 3: Real-time implementation scheme for the dual control strategy.

one of the smaller periods inside the first one.

\subsection{Commutation Protocol}

Once the period for all devices is detected, a supervision protocol should start to run with the aim to detect the event that enables the control commutation to the predictive control mode. During the autonomous control mode the optimal sequences previously determined are sent to the process and, the operational constraints are verified for the current instants to guarantee that the system keeps in its feasible region. However, if there is some change in the system or any disturbance takes place, then the system could reach a state of higher energy consumption or even an infeasible state. In the worse case, if there is some abnormal behaviour of the system and the activation/deactivation sequence of peripheral devices is not updated at the suitable time instant, the system state might violate the boundary of the feasible region. If infeasibility occurs, it will not be possible to come back to the first control mode, such as the optimisation problem behind the controller design will be infeasible for the current state of the process line. Therefore, the supervision module needs to predict the system behaviour in order to anticipate these undesirable behaviours based on the current state of the process line and the optimal sequences found in the first control mode.

In this regard, whenever the autonomous mode is running, a prediction of the behaviour of the process line is performed taking into account the models for both machines and peripheral devices and the current measurements from the available sensors. In this case, the prediction horizon has the same length as for the predictive control mode, i.e., $H_{p, c}=28 \mathrm{~s}$. Based on the system prediction, it is verified that the process variables $q_{j}$ remain inside its boundaries $\bar{q}_{j}$ and $\underline{q}_{j}$, and that the the energy consumption for the current prediction does not exceed the optimal energy consumption found in the first control mode. Then, if for some time instants along the prediction horizon the variables $q_{j}$ are out of their boundaries or the energy consumption along the prediction horizon surpasses a percentage $\eta$ of the optimal energy consumption, the commutation indicators will be activated at the current instant $k$ for switching to the first control mode at the instant $k+1$. Thus, the commutation protocol from the autonomous mode to the predictive control mode is based on a prediction of the behaviour of the process plant to guarantee that the dynamics of peripheral devices remain inside their feasible region and the energy consumption is not increasing. It should be noted that the commutation indicators are flag variables designed based on the results obtained from the first control mode, which correspond to the optimal values used for the comparison in the autonomous mode. These indicators are defined as

$$
\begin{gathered}
\text { If } q_{j} \notin\left[\bar{q}_{j}, \underline{q}_{j}\right] \text { then } \omega_{q_{j}}=1, \\
\text { If } \tilde{S} \geq \eta S^{*} \text { then } \omega_{S}=1,
\end{gathered}
$$

with $\tilde{S}$ the energy consumption along the prediction horizon, $S^{*}$ the optimal energy consumption, and $\omega_{Q}=\left[\omega_{q_{1}}, \cdots, \omega_{q_{j}}\right]^{T}$ and $\omega_{S}$ the commutation indicators.

\section{Simulation results}

\subsection{Energy consumption models}

To identify energy consumption models of both machines and peripheral devices, different sequences of $\boldsymbol{\Lambda}_{\mathbf{P}}$ and $\boldsymbol{\Lambda}_{\mathbf{M}_{\mathbf{i}}}$ were tested in a test bench that emulate the energy consumption of machine tools and their peripheral devices with the aim to obtain a rich range of the corresponding outputs $S_{P_{j}}$ and $S_{M_{i}}$. Subsequently, the energy consumption models for the three machines and the three peripheral devices in the process line were identified by using the n4sid routine of the System Identification Toolbox ${ }^{\mathrm{TM}}$ provided by Matlabß. In this regard, different values of $N$ were tested to identify the suitable matrices $A, B, C$, and $D$, which allow the highest fitting degree between the real and modelled outputs.

In Table 2, the model order and the fitting percentages between the model and real outputs are presented for each one of the machines and peripheral devices. It should be noted that the 
Table 2: Model order and fitting percentage for the energy consumption models identified by SI methods.

\begin{tabular}{ccc}
\hline Component & N & \% fitting \\
\hline$M_{1}$ & 5 & 94.83 \\
$M_{2}$ & 5 & 95.11 \\
$M_{3}$ & 5 & 94.83 \\
$P_{G, 1}$ & 3 & 74.96 \\
$P_{L, 1}$ & 4 & 60.27 \\
$P_{G, 2}$ & 2 & 90.37 \\
\hline
\end{tabular}

energy consumption models of machines were identified only for a machine cycle of duration $T_{M_{i}}$ using the sequence $\boldsymbol{\Lambda}_{\mathbf{M}_{\mathbf{i}}}$ as input data. Then, due to the periodic behaviour of machine tools and according to Assumption 1, the resulting energy consumption profile will be periodic and constant over time. Therefore, only the obtained matrices for the energy consumption models related to peripheral devices were considered and presented below:

$$
\begin{aligned}
& A_{G, 1}=\left[\begin{array}{ccc}
0.4498 & 0.03599 & 0.00118 \\
-0.8389 & 0.3033 & -0.05152 \\
-0.1222 & 0.1909 & 0.7697
\end{array}\right], \\
& B_{G, 1}=\left[\begin{array}{cc}
0.06329 & -0.04165 \\
-0.05235 & 0.0655 \\
-1.536 & 1.183
\end{array}\right] \text {, } \\
& C_{G, 1}=\left[\begin{array}{lll}
1650 & -478.3 & -75.28
\end{array}\right] \text {, } \\
& A_{L, 1}=\left[\begin{array}{cccc}
0.269 & 0.118 & 0.1127 & 0.0137 \\
-0.3314 & 0.5097 & 0.1162 & -0.0414 \\
-0.8701 & -0.0211 & 0.5067 & 0.04843 \\
0.1377 & -0.2754 & 1.287 & -0.4221
\end{array}\right] \text {, } \\
& B_{L, 1}=\left[\begin{array}{cc}
-0.1227 & 0.1363 \\
0.1775 & -0.07527 \\
-0.1427 & 0.117 \\
0.2009 & 0.2937
\end{array}\right], \\
& C_{L, 1}=\left[\begin{array}{llll}
-779.7 & 988.1 & 289.8 & -32.09
\end{array}\right] \text {, } \\
& A_{G, 2}=\left[\begin{array}{cc}
0.6217 & 0.07028 \\
0.08679 & 0.9798
\end{array}\right], \\
& B_{G, 2}=10^{-4}\left[\begin{array}{ll}
3.368 & -0.771
\end{array}\right]^{T}, \\
& C_{G, 2}=\left[\begin{array}{ll}
529.8 & -31.4
\end{array}\right],
\end{aligned}
$$

\subsection{Dual mode control strategy}

In order to test the performance of the proposed control strategy to improve the energy efficiency of manufacturing systems in flexible scenarios, the following production programs are considered:

- Production program $1\left(P P_{1}\right)$ : In this program only the two global peripheral devices are required since the installed capacity is enough to supply the resources consumption of machines in the process line. It means that during the execution of $P P_{1}$, the sequences $u_{L_{1}}\left\{1: H_{p}\right\}$ and $A p_{1}\left\{1: H_{p}\right\}$ should be null, and added to the optimisation problem in (13) into the set of constraints. Besides, the energy consumption model and the process dynamics related to the device $L_{1}$ will not be considered into the set of constraints since this device remains inactive during the execution of $P P_{1}$. This production program will be executed during 800 $\mathrm{s}$, and then, it is assumed that a change in the production program occurs since a new piece will be processed. The resources consumption of both compressed air and coolant of machines along $\boldsymbol{\Lambda}_{\mathbf{M}_{\mathbf{i}}}$ during this program is shown in Figure 2.

- Production program $2\left(P_{2}\right)$ : During this program, the consumption of compressed air from $M_{1}$ is increased from $0.0015 \frac{\mathrm{kg}}{\mathrm{s}}$ to $0.003 \frac{\mathrm{kg}}{\mathrm{s}}$ while keeping the same for the other two machines in the process line. Since the maximum capacity of the global peripheral device $G_{1}$ when is turned on is $0.006 \frac{\mathrm{kg}}{\mathrm{s}}$, it is not able to provided the air stream required by all the machines in the process line, and therefore the local device $L_{1}$ should be activated to help supplying the air stream required by the first machine. The pipe system that connects $L_{1}$ to $M_{1}$ to allow a maximum flow of $0.003 \frac{\mathrm{kg}}{\mathrm{s}}$, while the maximum flow thorough the connection between $G_{1}$ and $M_{1}$ remains equal to $0.0015 \frac{\mathrm{kg}}{\mathrm{s}}$ as for the first program. In addition, when $P P_{2}$ is running, the dynamics for the device $L_{1}$ and its energy consumption model should be considered into the set of constraints of the optimisation problem in (13). It should be noted that the resource consumption for the rest of machines in the process line keep the same as for $P P_{1}$ as shown in Figure 2.

Based on the previous description of production programs, $P P_{1}$ was executed during the first 800 s of simulation, and then, the production program was commuted to $P P_{2}$. In this case, simulations along $2000 \mathrm{~s}$ were performed using a execution time of the controller equals $1 \mathrm{~s}$. This latter fact means that the controller makes decision every second along the prediction horizon, and the required information for the controller is also updated every second before to run the controller again. All simulations were performed using an Intel Core i7-55000U $2.4 \mathrm{GHz}$ processor with $8 \mathrm{G}$ RAM and considering a sampling time $\tau_{s}=0.1 \mathrm{~s}$. The simulation results were obtained in Matlab by using the software IBM ILOG CPLEX Optimisation Studio [7] integrated to YALMIP toolbox [8]. Moreover, according to Algorithm 1, the xcorr routine of Matlab was used to compute the autocorrelation $\mathbf{u x}_{\mathbf{j}}$ of the pre-processed signal of $\hat{\mathbf{u}}_{j}$, from which were obtained the vectors of both correlation coefficients and lags.

The obtained optimal activation sequences for both peripheral devices and the percentage of aperture valve for $v_{1}$ and $v_{3}$ are shown in Figures $4 \mathrm{a}$ and $4 \mathrm{~b}$, respectively. According to these results, the device $L_{1}$ is activated only when $P P_{2}$ is executed, and the periodic behaviour of all devices in the process line can be observed. In Figure $4 a$, the time slots in which the predictive control mode is running are marked, while for the rest of the time the controller is on the autonomous mode. 
When the controller is in the autonomous mode, the periodicity of each device has been already determined during the predictive mode, and therefore, the periodic behaviour in the activation sequence is also appreciated in the final part of the predictive control mode. It should be noted that, only during the predictive control mode the controller solves the optimisation problem in (13), and in the autonomous mode, the periodic sequences are sent to the plant and only it is verified that the system remains inside the feasible domain found in the optimisation-based mode. The latter procedure corresponds to the communication protocol.

Besides, concerning the change in the production program, from Figure $4 \mathrm{~b}$, it can observe that since the energy consumption of $G_{1}$ is higher than the energy consumption of $L_{1}$, the controller decides supplying all the compressed air required by $M_{1}$ from $L_{1}$ reducing the demand for $G_{1}$, and allowing this latter device remains turned on for less time to minimise the global energy consumption. In this regard, $L_{1}$ is responsible for providing all the demand of $M_{1}$, while $v_{1}$ is totally closed and $G_{1}$ supplies only to machines $M_{2}$ and $M_{3}$. Next, according to the obtained activation sequences, in Figures $5 \mathrm{a}$ and $5 \mathrm{~b}$, the resulting energy consumption of the process line and the particular energy consumption of each device obtained when the proposed control strategy is implement are presented, respectively. From these results, it is also possible to see that the peripheral device $L_{1}$ starts to consume energy only when the second production program is activated, and therefore, the global energy consumption of the process line increases after this moment.

In the same way, in Figures $6 a$ and $6 b$, the dynamics for the supply systems of compressed air and coolant associated to peripheral devices in the process line are presented. Based on these results, it can be seen that both the pressure and level dynamics remain inside their upper and lower bounds while either of the production programs is executed. Besides, the periodic behaviour can also be detected from these dynamics (for each production program) after the predictive control mode is activated. Thus, due to the nature of these dynamics, both pressure and level dynamics were employed to check the periodicity condition as shown in Algorithm 1. The last fact is because when using the activation sequences of peripheral devices, which are usually constrained to $\{0,1\}$, the periodicity condition could be verified for values of $T_{u_{j}}$ that are not the real period for the devices. Then, based on the obtained results for the optimal sequences of $u_{j}$ and process dynamics $q_{j}$, the values of $T_{u_{G_{1}}}$ and $T_{u_{G_{2}}}$ detected while $P P_{1}$ was ran were equal to 140 and $84 \mathrm{~s}$, respectively. For the case in which $P P_{2}$ was executed, the periods identified for $G_{1}, G_{2}$ and $L_{1}$ were 28,84 and $28 \mathrm{~s}$, respectively.

Next, to verify the computational burden and the suitability to implement the proposed control strategy in real time, the computational time spent by iteration is presented in Figure 7. Based on these results, it can be seen that even when the predictive control mode is on, the maximum time spent is lower than one second. Besides, it should be noted that when the autonomous mode is on, the computational time significantly decreases since, in this mode, it should not solve an optimisation problem. Thus, the time spent in the autonomous mode
Table 3: Performance degradation with respect to a conventional MPC.

\begin{tabular}{ccc}
\hline Controller & Energy consumption & Simulation time \\
\hline MPC & $3299596.872 \mathrm{VA}$ & $169.470 \mathrm{~s}$ \\
Dual control & $3299578.348 \mathrm{VA}$ & $60.681 \mathrm{~s}$ \\
\hline
\end{tabular}

refers to the time required for the commutation protocol to predict the system behaviour and to verify that the system remains into its feasible domain for the whole prediction. Then, according to these results, it is possible to conclude that the proposed control strategy is suitable to be implemented in real time.

\subsection{Performance degradation}

In this section, the proposed control strategy based on two different control modes is compared with a conventional MPC controller with the aim to evaluate the performance degradation when a periodic behaviour is detected and the autonomous mode is switched on. In this regard, a predictive-like controller based on the MPC approach and the same optimisation problem in (13) was designed and used to determine the optimal activation sequences of peripheral devices when the optimisation problem is solved during the whole simulation. The MPC controller was tested at the same operational conditions as for the dual control mode strategy and considering the same flexibility scenario presented in the previous section.

The obtained results for energy consumption profile are presented in Figure 8a and summarised in Table 3. From these results, it is possible to observe that the main differences in the activation sequences of peripheral devices are related to the device $G_{2}$, for which the activation load can be modulated. These small variations in the modulation of $u_{G_{2}}$ could be a consequence of the termination criteria defined by default for the solver cplex and the current state of the system. That means, depending on the current state of the system, i.e., the current state of energy consumption models and process dynamics of peripheral devices, the termination criteria might be achieved at different values of the feasible domain for the decision variables. Thus, regarding the performance of control strategies in closed-loop, the degradation of the performance when the proposed dual control strategy is implemented with respect to a control strategy that is constantly optimising online is almost none, with differences near $8.7 \times 10^{-4}$. However, regarding the computational burden, when the system exhibits a periodic behaviour and it is used to commute to autonomous mode, the time spent is significantly lower and the possibilities to implement the strategy in real time increases. Besides, by implementing the commutation protocol during the autonomous mode to check that the system remains inside its feasible domain, the computational burden is reduced without affecting significantly the effectiveness of the control strategy.

\subsection{Comparative assessment and disturbances management}

In this section, the performance of the proposed dual control mode strategy is compared with an MPC controller that is constantly optimising for the whole simulation when disturbances take place and affect the operation of the process line. 

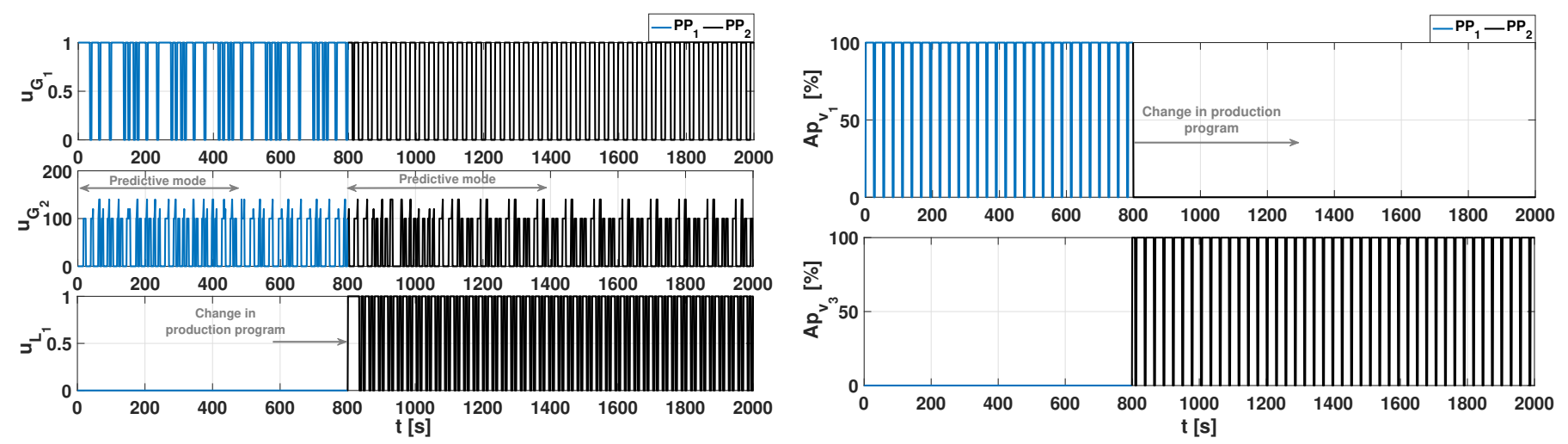

(a) Optimal activation sequences of peripheral devices in the process line.

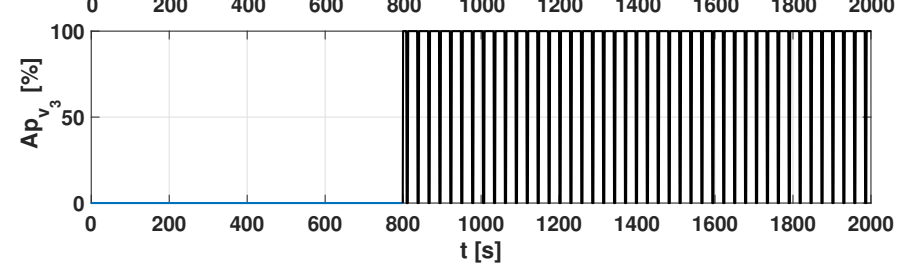

(b) Optimal sequences of valve aperture for $v_{1}$ and $v_{3}$.

Figure 4: Optimal activation sequences of peripheral devices by using the proposed control strategy.

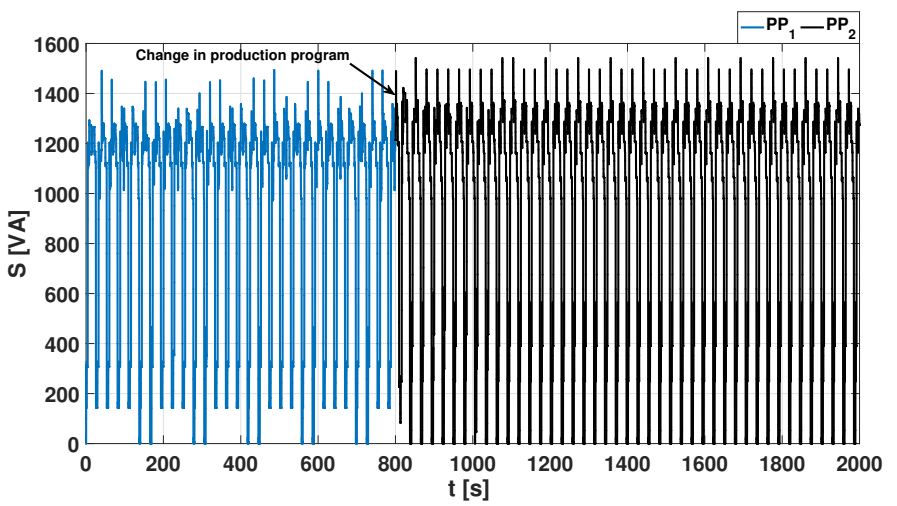

(a) Profile of the total energy consumption of the process line.

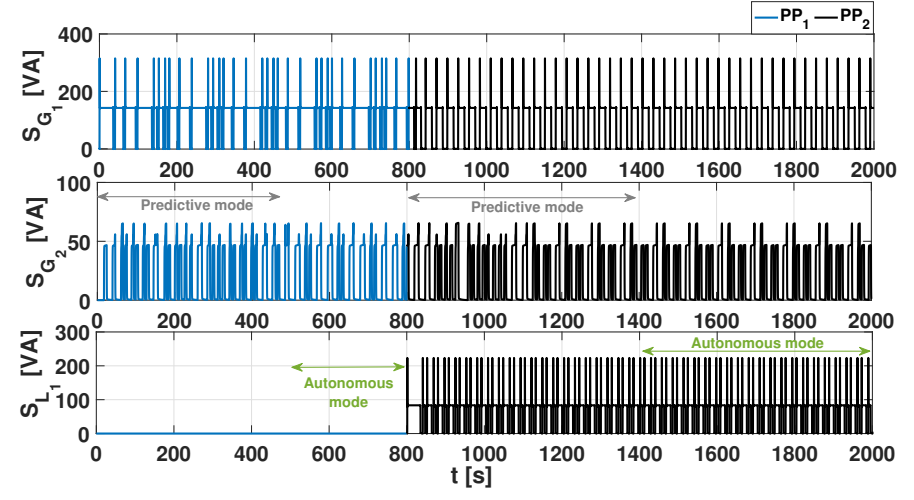

(b) Energy consumption profile of devices $G_{1}, G_{2}$ and $L_{1}$, respectively.

Figure 5: Energy consumption profiles by using the proposed control strategy.
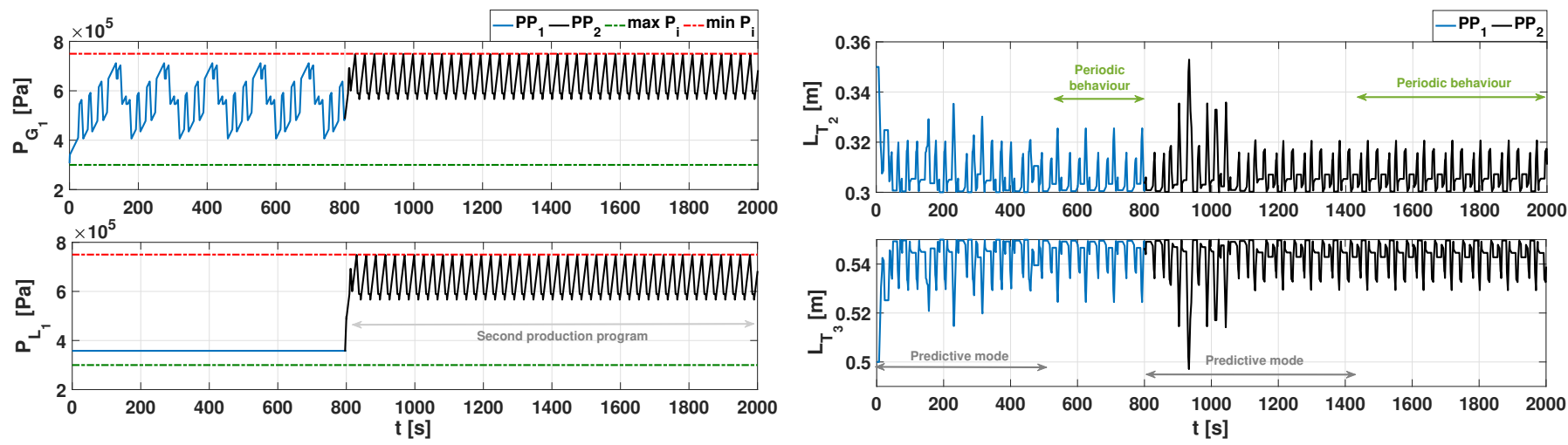

(b) Level dynamics for both clean and dirty coolant tanks.

(a) Pressure dynamics for both $L_{1}$ and $G_{1}$.

Figure 6: Process dynamics related to the operation of peripheral devices.

The underlying idea of this comparative study is to understand how conservative the dual control strategy should be before to commute to the predictive control mode when some disturbance happens. As explained in Section 4.2, a prediction of $H_{p, c}=28$ $\mathrm{s}$ is made at each iteration while the autonomous control mode is running, and, if it detects that the system reaches out of its feasible domain (determined from the predictive control mode) in any moment along the prediction horizon, the system should commute to the predictive control mode. That means if, at the current instant, the prediction shows that at the instant $t=28$ $\mathrm{s}$, the systems surpasses some of its boundaries, the control commutes. However, this is the most conservative possible scenario, since for the case in which the disturbance persists, for instance along 3 seconds from the current instant and then disappears, the control mode switching would be unnecessary, and the computational burden increases. In this regard, the proposed control strategy is tested considering scenarios with disturbances and different values of $H_{p, c}$ to evaluate its effec- 


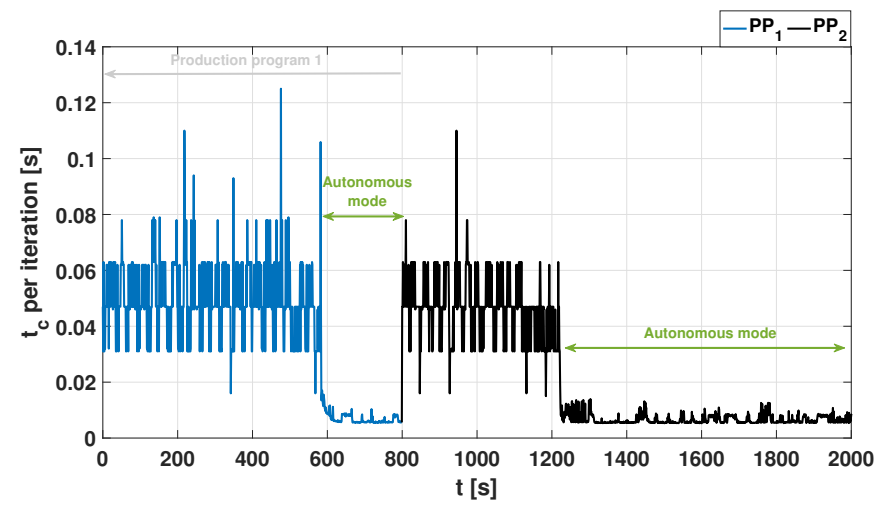

Figure 7: Real-time implementation scheme for the dual control strategy.

Table 4: Comparative assessment for different values of $H_{p, c}$.

\begin{tabular}{cccc}
\hline$H_{p, c}$ & Controller & Energy consumption & Simulation time \\
\hline \multicolumn{4}{c}{ Disturbance 1 } \\
\hline- & MPC & $1363532.825 \mathrm{VA}$ & $77.139 \mathrm{~s}$ \\
$28 \mathrm{~s}$ & Dual control & $1363447.325 \mathrm{VA}$ & $84.265 \mathrm{~s}$ \\
$14 \mathrm{~s}$ & Dual control & 1363447.325 VA & $74.062 \mathrm{~s}$ \\
$7 \mathrm{~s}$ & Dual control & Infeasible & - \\
\hline \multicolumn{4}{c}{ Disturbance 2 } \\
\hline- & MPC & $1364306.771 \mathrm{VA}$ & $83.680 \mathrm{~s}$ \\
$28 \mathrm{~s}$ & Dual control & $1363872.542 \mathrm{VA}$ & $50.975 \mathrm{~s}$ \\
$14 \mathrm{~s}$ & Dual control & $1364225.510 \mathrm{VA}$ & $50.530 \mathrm{~s}$ \\
$7 \mathrm{~s}$ & Dual control & $1364325.510 \mathrm{VA}$ & $51.643 \mathrm{~s}$ \\
\hline
\end{tabular}

tiveness regarding the conventional MPC. Then, based on the obtained results, the suitable length of $H_{p, c}$ can be determined, from which a proper performance can be achieved without increasing the computational burden.

Two different disturbances were considered and three different lengths of $H_{p, c}$ were tested in order to compare the performance and computational burden of the proposed control strategy with respect to the conventional MPC. For the former, a fault in the device $G_{2}$ is assumed, considering a reduction of its efficiency from $95 \%$ to $75 \%$ along $25 \mathrm{~s}$. Thus, the real flow of coolant pumps from $T_{3}$ to $T_{2}$ will be lower that the flow considered into the control-oriented model (in the controller design). The second disturbance is applied to modify the operation of $G_{1}$ and emulates a fault of its operation. Thus, an air leak equal $0.0023 \frac{\mathrm{kg}}{\mathrm{s}}$ was added during $25 \mathrm{~s}$. Based on the previous description of the considered disturbances, different simulations using the proposed dual control mode switching strategy were performed modifying the value of $H_{p, c}$ to compare both the resulting energy consumption and the time spent in the simulations with respect to the control strategy that is constantly optimising. The obtained results for the total energy consumption along $1400 \mathrm{~s}$ and the total simulation time are presented in Table 4 .

Based on the obtained results, it is possible to conclude that for choosing the length of $H_{p, c}$ both the nature of the disturbances applied as well as its duration should be considered. However, to analyse most of the possible situations, in magnitude and duration, is a tedious task. For the case of disturbance
1 , the first time that the commutation protocol detects that the system will be outside of its feasible domain corresponds to the instant $13 \mathrm{~s}$ in the prediction horizon. Thus, for lengths of $H_{p, c}$ shorter than nine seconds the commutation protocol was not able to detect that the system will violate the boundary of the feasible domain well in advance. Therefore, the optimisation problem in the second mode was infeasible at the time in which the commutation protocol decided to switch to the first control mode. Regarding the second disturbance, its effects on the operation of peripheral devices were only observed after the disturbance disappears. Thus, when the controller switched to the predictive control mode, the system is at the normal operational conditions again, and the MPC can reach faster the periodic behaviour. Therefore, in contrast to the scenario of the first disturbance, in this case, the length of $H_{p, c}$ was not critical since when the disturbance was detected, there were not unexpected behaviours that try to move the system outside of its feasible domain. In this regard, when selecting $H_{p, c}$ in the commutation protocol equals to $H_{p}$ for the first control mode, it is possible to avoid the system to reach infeasible domains while the autonomous mode is running even when disturbances take place. Besides, according to the results in Table 4, the performance of the proposed control strategy is not significantly improved if the length of $H_{p, c}$ is shorter.

\section{Conclusion}

In this paper, a dual control mode switching strategy is proposed to improve the energy efficiency of complex manufacturing systems without affecting their productivity. In this regard, machines in the process line and their corresponding energy consumption were assumed constant over the time to process the same number of pieces as when the control strategy is not implemented. Thus, the strategy was designed based on two control modes. In the former, a predictive-like controller is executed up to a periodic behaviour of the optimised system is detected (if such behaviour occurs). Then, once the periodicity is detected, the system commutes to an autonomous control mode to reduce the computational burden. Due to the nature of system actuators, the predictive-like controller is designed based on a mixed-integer linear programming problem, in which both energy consumption models and the process dynamics performed by peripheral devices are included in the constraints set.

According to the obtained results, the proposed control strategy allows managing the peripheral devices to reduce the global energy consumption and reducing the computational burden without decreasing the productivity of the process line, even when flexible-manufacturing scenarios are considered. Thus, given the reduction in computational load, the proposed control strategy is suitable to be implemented in real time even when the number of decision variables increases, such as in the case of complex, large-scale, and flexible manufacturing systems. Besides, the proposed strategy is able to manage disturbances in the operation of peripheral devices, even when the autonomous mode is running, if the commutation protocol is suitably designed to switch fast enough to the predictive control mode and to avoid that the system reaches infeasible states. 


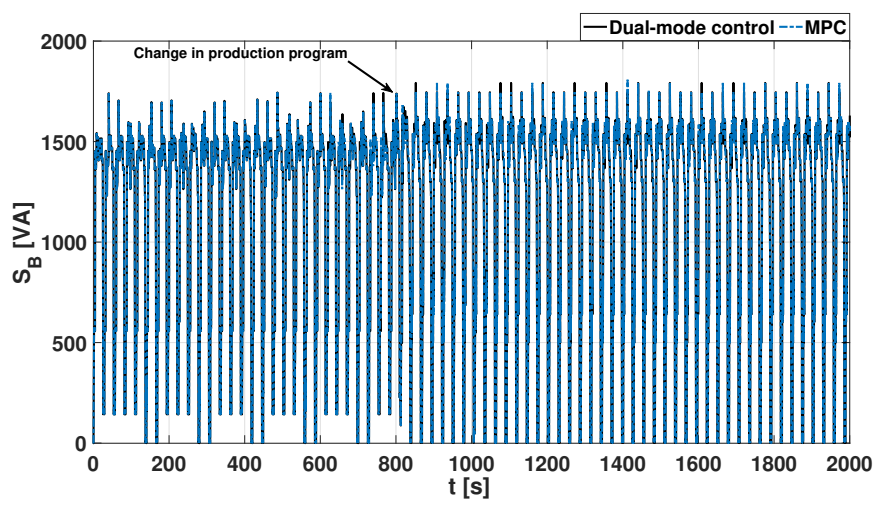

(a) Profile of the total energy consumption.

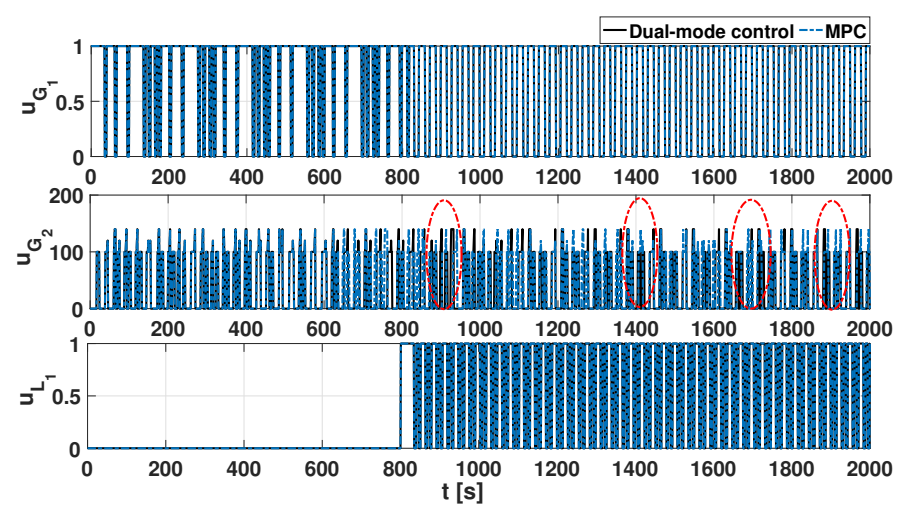

(b) Optimal activation sequences of devices $G_{1}, G_{2}$ and $L_{1}$.

Figure 8: Simulation results of the comparison between the dual control mode strategy and the conventional MPC.

In this regard, the proposed dual control mode strategy could be tested using decentralised control architectures to test its effectiveness and to compare how faster the periodicity for the activation signal could be detected using modular (or smaller) control loops. Besides, taking advantage of the commutation protocol to determine when the difference between the predicted energy consumption and the optimal energy consumption increases, it can be possible to decide when the energy consumption models should be re-identified. Also, taking into account that the energy consumption of manufacturing systems can change due to the wear or damage in either machines or devices, maintenance tasks could be scheduled well in advanced to minimise the adverse effects over the plant productivity.

\section{Acknowledgments}

This work has been partially funded by the project IKERCON (ref. C10683) and and the FI-AGAUR scholarship of the Catalan government.

\section{References}

[1] E. Abele, N. Panten, and B. Menz. Data collection for energy monitoring purposes and energy control of production machines. Procedia CIRP, 29:299-304, 2015. The 22nd CIRP Conference on Life Cycle Engineering.

[2] A.A.G. Bruzzone, D. Anghinolfi, M. Paolucci, and F. Tonelli. Energyaware scheduling for improving manufacturing process sustainability: A mathematical model for flexible flow shops. CIRP Annals, 61(1):459462, 2012.

[3] J. L. Diaz C. and C. Ocampo-Martinez. Energy efficiency in discretemanufacturing systems: Insights, trends, and control strategies. Journal of Manufacturing Systems, 52:131-145, 2019.

[4] H.A. ElMaraghy, A.M.A. Youssef, A.M. Marzouk, and W.H. ElMaraghy. Energy use analysis and local benchmarking of manufacturing lines. Journal of Cleaner Production, 163:36-48, 2017.

[5] B. Esmaeilian, S. Behdad, and B. Wang. The evolution and future of manufacturing: A review. Journal of Manufacturing Systems, 39:79-100, 2016.

[6] Z. Gajic. Linear dynamic systems and signals. Prentice Hall/Pearson Education, Upper Saddle River, N.J, 2003.

[7] IBM. IBM ILOG CPLEX optimization studio, v12. 5, 2013.

[8] J. Löfberg. Yalmip : A toolbox for modeling and optimization in Matlab. In In Proceedings of the CACSD Conference, Taipei, Taiwan, 2004.
[9] C. Lu, L. Gao, X. Li, Q. Pan, and Q. Wang. Energy-efficient permutation flow shop scheduling problem using a hybrid multi-objective backtracking search algorithm. Journal of Cleaner Production, 144:228-238, 2017.

[10] J.M. Maciejowski. Predictive Control with Constraints. Prentice Hall, Great Britain, 2002.

[11] P. Overschee and B. De Moor. Subspace Identification for Linear Systems: Theory - Implementation - Applications. Springer US, Boston, MA, 1996.

[12] C.T. Papadopoulos, J. Li, and M.E.J. O'Kelly. A classification and review of timed markov models of manufacturing systems. Computers $\mathcal{F}$ Industrial Engineering, 128:219-244, 2019.

[13] S.J. Qin. An overview of subspace identification. Computers $\mathcal{E}$ Chemical Engineering, 30(10):1502-1513, 2006. Papers form Chemical Process Control VII.

[14] J.B. Rawlings and D.Q. Mayne. Model Predictive Control: Theory and Design. Nob Hill Publishing, Madison, WI (USA), 2009.

[15] M.U. Uluer, H.O. Unver, G. Gok, N. Fescioglu-Unver, and S.E. Kilic. A framework for energy reduction in manufacturing process chains (eMPC) and a case study from the turkish household appliance industry. Journal of Cleaner Production, 112:3342-3360, 2016.

[16] M. Verhaegen and A. Hansson. N2sid: Nuclear norm subspace identification of innovation models. Automatica, 72:57-63, 2016.

[17] J. Yan, L. Li, F. Zhao, F. Zhang, and Q. Zhao. A multi-level optimization approach for energy-efficient flexible flow shop scheduling. Journal of Cleaner Production, 137:1543-1552, 2016.

[18] R. Yin. Metallurgical process engineering. Metallurgical Industry Press Springer, Berlin, 2011.

[19] Z. Zhou, B. Yao, W. Xu, and L. Wang. Condition monitoring towards energy-efficient manufacturing: a review. The International Journal of Advanced Manufacturing Technology, 91(9-12):3395-3415, 2017. 